\title{
Scheme-independent series for anomalous dimensions of higher-spin operators at an infrared fixed point in a gauge theory
}

\author{
Thomas A. Ryttov ${ }^{1}$ and Robert Shrock $\oplus^{2}$ \\ ${ }^{1} C P^{3}$-Origins and Danish Institute for Advanced Study, Southern Denmark University, \\ Campusvej 55, Odense, Denmark \\ ${ }^{2}$ C. N. Yang Institute for Theoretical Physics and Department of Physics and Astronomy, \\ Stony Brook University, Stony Brook, New York 11794, USA
}

(Received 20 February 2020; accepted 8 April 2020; published 23 April 2020)

\begin{abstract}
We consider an asymptotically free vectorial gauge theory, with gauge group $G$ and $N_{f}$ fermions in a representation $R$ of $G$, having an infrared fixed point of the renormalization group. We calculate schemeindependent series expansions for the anomalous dimensions of higher-spin bilinear fermion operators at this infrared fixed point up to $O\left(\Delta_{f}^{3}\right)$, where $\Delta_{f}$ is an $N_{f}$-dependent expansion variable. Our general results are evaluated for several special cases, including the case $G=\mathrm{SU}\left(N_{c}\right)$ with $R$ equal to the fundamental and adjoint representations.
\end{abstract}

DOI: $10.1103 /$ PhysRevD.101.076018

\section{INTRODUCTION}

An asymptotically free gauge theory with sufficiently many massless fermions evolves from the deep ultraviolet (UV) to an infrared fixed point (IRFP) of the renormalization group at a zero of the beta function. The theory at this IRFP exhibits scale invariance due to the vanishing of the beta function. The properties of the theory at this IRFP are of fundamental field-theoretic interest. Among the basic properties are the anomalous dimensions $\gamma_{\mathrm{IR}}^{(\mathcal{O})}$ of various gauge-invariant operators $\mathcal{O}$.

In this paper we consider an asymptotically free vectorial gauge theory of this type, with a general gauge group $G$ and $N_{f}$ copies ("flavors") of massless Dirac fermions $\psi_{i}$, $i=1, \ldots, N_{f}$, transforming according to a representation $R$ of $G$ [1]. We present scheme-independent series expansions of the anomalous dimensions of gauge-invariant higher-spin operators that are bilinear in the fermion fields, up to $O\left(\Delta_{f}^{3}\right)$ inclusive, at the infrared fixed point, where $\Delta_{f}$ is an $N_{f}$-dependent expansion variable defined below, in Eq. (1.8). The operators that we consider are of the form (suppressing flavor indices) $\bar{\psi} \gamma_{\mu_{1}} D_{\mu_{2}} \ldots D_{\mu_{j}} \psi$ and $\bar{\psi} \sigma_{\lambda \mu_{1}} D_{\mu_{2}} \ldots D_{\mu_{j}} \psi$, where $D_{\mu}$ is the covariant derivative for the gauge theory, and it is understood here and below that the operators are symmetrized over the Lorentz indices $\mu_{i}, 1 \leq i \leq j$ and have Lorentz traces subtracted, and $\sigma_{\lambda \mu_{1}}$ is

Published by the American Physical Society under the terms of the Creative Commons Attribution 4.0 International license. Further distribution of this work must maintain attribution to the author(s) and the published article's title, journal citation, and DOI. Funded by SCOAP. the commutator of two Dirac matrices [defined in Eq. (2.3)]. We consider the cases $1 \leq j \leq 3$.

The operators $\bar{\psi} \gamma_{\mu_{1}} D_{\mu_{2}} \ldots D_{\mu_{j}} \psi$ were considered early on in the analysis of approximate Bjorken scaling in deep inelastic lepton scattering and the associated development of the theory of quantum chromodynamics (QCD). We briefly review this background [2-13]. In Euclidean quantum field theory, the short-distance operator product expansion (OPE) expresses the product of two operators $A(x)$ and $B(y)$ as a sum of local operators $\mathcal{O}_{i}$ multiplied by coefficient functions $c_{\mathcal{O}_{i}}$,

$$
A(x) B(y)=\sum_{i} c_{\mathcal{O}_{i}}(x-y) \mathcal{O}_{i}((x+y) / 2),
$$

in the limit where $x-y \rightarrow 0$. Let us denote the Maxwellian (i.e., free-field) dimension of an operator $\mathcal{O}$ in mass units as $d_{\mathcal{O}}$. Then the (free-field) dimension of the coefficient function is $d_{c_{\mathcal{O} i}}=d_{A}+d_{B}-d_{\mathcal{O}_{i}}$, so

$$
c_{\mathcal{O}_{i}}(x-y) \sim|x-y|^{d_{\mathcal{O}_{i}}-d_{A}-d_{B}},
$$

where $|x-y|$ refers to the Euclidean distance. Hence, in the short-distance OPE, the operators with the lowest dimensions dominate, since they are multiplied by the smallest powers of $|x-y|$. However, deep inelastic scattering and the associated Bjorken limit probe the light cone limit, $(x-y)^{2} \rightarrow 0$ with $x-y \neq 0$ in Minkowski space, where $x^{2}=x_{\mu} x^{\mu}$. With the arguments of two illustrative Lorentzscalar operators denoted in a symmetric manner as $\pm x / 2$, the light-cone OPE for $A(x / 2) B(-x / 2)$ is 


$$
A(x / 2) B(-x / 2)=\sum_{i, n} \bar{c}_{i, n}\left(x^{2}\right) x^{\mu_{1}} \cdots x^{\mu_{n}} \mathcal{O}_{i, n ; \mu_{1}, \ldots, \mu_{n}}(0)
$$

in the limit $x^{2} \rightarrow 0$, where the coefficient functions have been written in a form that explicitly indicates the factor $x^{\mu_{1}} \cdots x^{\mu_{n}}$ and the operator $\mathcal{O}_{i, n ; \mu_{1}, \ldots, \mu_{n}}$ has spin $j=n$. Here (suppressing the Lorentz indices on $\mathcal{O}_{i, n ; \mu_{1}, \ldots, \mu_{n}}$ ) the dependence of $\bar{c}_{i, n}$ on $x^{2}$ is

$$
\bar{c}_{i, n}\left(x^{2}\right) \sim\left(x^{2}\right)^{\left(d_{\mathcal{O}_{i, n}}-n-d_{A}-d_{B}\right) / 2}
$$

(with logarithmic corrections in QCD due to anomalous dimensions). Consequently, the operators that have the strongest singularity in their coefficient function $\bar{c}_{i, n}\left(x^{2}\right)$ as $x^{2} \rightarrow 0$ and hence make the dominant contribution to the right-hand side of the light-cone OPE, Eq. (1.3) are those with minimal "twist" $\tau$ [7], where $\tau$ is the dimension minus the spin $j$ of the operator, i.e.,

$$
\tau_{\mathcal{O}_{i, n}}=d_{\mathcal{O}_{i, n}}-j_{\mathcal{O}_{i, n}}
$$

with $j_{\mathcal{O}_{i, n}}=n$ here. Thus, among bilinear fermion operators, in addition to $\bar{\psi} \gamma_{\mu_{1}} \psi$ with dimension 3, spin 1, and hence $\tau=2$, there are the operators $\bar{\psi} \gamma_{\mu_{1}} D_{\mu_{2}} \cdots D_{\mu_{j}} \psi$, with dimension $3+(j-1)$ and spin $j$, which also have $\tau=2$. These are the minimum-twist bilinear fermion operators that contribute to the light-cone OPE (1.3) [14]. In a similar manner, twist-2 operators make the dominant contribution to the right-hand side of the light-cone OPE for the product of two electromagnetic or weak currents. The other operators that we consider, namely $\bar{\psi} \sigma_{\lambda \mu_{1}} D_{\mu_{2}} \ldots D_{\mu_{j}} \psi$, have been relevant for the study of transversity distributions in QCD [15].

Our approach here is complementary to these previous analyses of higher-spin operators, which have focused on applications to QCD. In contrast, we study the anomalous dimensions of these operators at an infrared fixed point in a (deconfined) chirally symmetric non-Abelian Coulomb phase (NACP), where the theory is scale invariant and is inferred to be conformally invariant [16], hence the commonly used term "conformal window." The goal of our calculations is to gain information about the properties of the conformal field theory that is defined at this IRFP.

Let us recall some further relevant background for our work. The evolution of the running gauge coupling $g=g(\mu)$, as a function of the momentum scale, $\mu$, is described by the renormalization-group (RG) beta function $\beta=d \alpha / d \ln \mu$, where $\alpha(\mu)=g(\mu)^{2} /(4 \pi)$. From the oneloop term in the beta function $[10,11]$, it follows that the property of asymptotic freedom restricts $N_{f}$ to be less than an upper $(u)$ bound, $N_{u}$, where [17]

$$
N_{u}=\frac{11 C_{A}}{4 T_{f}} .
$$

Here, $C_{A}$ is the quadratic Casimir invariant for the group $G$ and $T_{f}$ is the trace invariant for the representation $R$ [18]. If $N_{f}$ is slightly less than $N_{u}$, then this theory has an infrared zero in the (perturbatively calculated) beta function, i.e., an IR fixed point of the renormalization group, at a value that we shall denote $\alpha_{\mathrm{IR}}[19,20]$. In the two-loop beta function (with $N_{f}<N_{u}$ as required by asymptotic freedom), this IR zero is present if $N_{f}$ is larger than a lower $(\ell)$ value $N_{\ell}$, where [19]

$$
N_{\ell}=\frac{17 C_{A}^{2}}{2 T_{f}\left(5 C_{A}+3 C_{f}\right)} .
$$

As the scale $\mu$ decreases from large values in the UV to small values in the IR, $\alpha(\mu)$ approaches $\alpha_{\mathrm{IR}}$ from below as $\mu \rightarrow 0$. Here we consider the properties of the theory at this IRFP in the perturbative beta function. (For a discussion of an IR zero in a nonperturbatively defined beta function and its application to QCD, see [21].)

Since the anomalous dimensions of gauge-invariant operators evaluated at the IRFP are physical, they must be independent of the scheme used for regularization and renormalization. In the conventional approach, one first expresses these anomalous dimensions as series expansions in powers of $\alpha$ or equivalently $a=g^{2} /\left(16 \pi^{2}\right)=\alpha /(4 \pi)$, calculated to $n$-loop order; second, one computes the IR zero of the beta function, denoted $\alpha_{\mathrm{IR}, n}$, to the same $n$-loop order; and third, one sets $\alpha=\alpha_{\mathrm{IR}, n}$ in the series expansion for the given anomalous dimension to obtain its value at the IR zero of the beta function to this $n$-loop order. For the operator $\bar{\psi} \psi$ this conventional approach to calculate anomalous dimensions at an IR fixed point was carried out to the four-loop level in [22-24] and to the five-loop level in [25]. However, these conventional series expansions in powers of $\alpha$, calculated to a finite order, are scheme-dependent beyond the leading terms. This is a wellknown property of higher-order QCD calculations used to fit actual experimental data, which, in turn, has motivated many studies to reduce scheme dependence [26]. These studies dealt with the UV fixed point (UVFP) at $\alpha=0$, as is appropriate for QCD. Studies of scheme dependence of quantities calculated in a conventional manner at an IR fixed point at $\alpha_{\mathrm{IR}}$ were carried out in [27-31]. In particular, it was shown that many scheme transformations that are admissible in the vicinity of the UVFP at $\alpha=0$ in an asymptotically free theory are not admissible away from the origin because of various pathological properties. For example, the scheme transformation $r a=\tanh \left(r a^{\prime}\right)$ (depending on a parameter $r$ ) is an admissible transformation in the neighborhood of $\alpha=\alpha^{\prime}=0$. However, the inverse of this transformation is $a^{\prime}=(2 r)^{-1} \ln [(1+r a) /$ $(1-r a)]$, which is singular at an IRFP with $a_{\mathrm{IR}} \geq 1 / r$, i.e., 
$\alpha_{\mathrm{IR}} \geq 4 \pi / r$, so that the transformation is not admissible at this IRFP. References [27] derived and studied an explicit scheme transformation that removes terms of loop order 3 and higher from the beta function in the local vicinity of $\alpha=0$, as is relevant to the UVFP in QCD [32], but also showed that such a scheme transformation cannot, in general, be used at an IRFP away from the origin owing to various pathologies, one of which was illustrated above.

It is thus desirable to use a theoretical framework in which the series expansions for physical quantities, such as anomalous dimensions of gauge-invariant operators at the IRFP, are scheme-independent at any finite order in an expansion variable. Because $\alpha_{\mathrm{IR}} \rightarrow 0$ as $N_{f}$ approaches $N_{u}$ from below (where $N_{f}$ is formally generalized here from a non-negative integer to a non-negative real number [17]), one can reexpress the expansions for physical quantities at the IRFP as power series in the manifestly schemeindependent quantity $[20,33]$

$$
\Delta_{f}=N_{u}-N_{f} .
$$

In previous work we have calculated scheme-independent expansions for anomalous dimensions of several types of gauge-invariant operators at an IRFP in an asymptotically free gauge theory [34-40]. We have compared the resultant values for anomalous dimensions with lattice measurements where available [35-37,41,42].

In the present paper we extend these calculations to the case of the higher-spin operators $\bar{\psi} \gamma_{\mu_{1}} D_{\mu_{2}} \ldots D_{\mu_{j}} \psi$ and $\bar{\psi} \sigma_{\lambda \mu_{1}} D_{\mu_{2}} \ldots D_{\mu_{j}} \psi$ for $1 \leq j \leq 3$. In addition to general formulas, we present results for several different special cases, including the case where $G=\mathrm{SU}\left(N_{c}\right)$ and the fermions are in the fundamental $(F)$ and adjoint $(A d j)$ representations. We also give results for the limit $N_{c} \rightarrow \infty$ and $N_{f} \rightarrow \infty$ with the ratio $N_{f} / N_{c}$ fixed and finite. Our calculations show that these scheme-independent expansions of the anomalous dimensions of the operators are reasonably accurate throughout much of the non-Abelian Coulomb phase. Our results give further insight into the properties of a theory at an IRFP and should be useful to compare with lattice measurements of the anomalous dimensions of these higher-spin operators when such measurements will be performed [43].

This paper is organized as follows. Some relevant background and methods are discussed in Sec. II. General structural forms for the anomalous dimensions of higher-spin bilinear fermion operators are given in Sec. III. In Sec. IV we present our scheme-independent calculations of the anomalous dimensions of these higherspin Wilson operators for a general gauge group $G$ and fermion representation $R$. In Sec. V we give results for the case where $G=\mathrm{SU}\left(N_{c}\right)$ and $R$ is the fundamental representation, and in Sec. VI we present the special case of these results for the limit $N_{c} \rightarrow \infty$ and $N_{f} \rightarrow \infty$ with $N_{f} / N_{c}$ fixed and finite. Anomalous dimension calculations for the case where $G=\mathrm{SU}\left(N_{c}\right)$ and $R$ is the adjoint representation are presented in Sec. VII. Our conclusions are given in Sec. VIII and some auxiliary results are included in Appendix.

\section{CALCULATIONAL METHODS}

Let us consider a (gauge-invariant) operator $\mathcal{O}$. Because of the interactions, the full scaling dimension of this operator, denoted $D_{\mathcal{O}}$, differs from its free-field value, $D_{\mathcal{O}, \text { free }} \equiv d_{\mathcal{O}}$ :

$$
D_{\mathcal{O}}=D_{\mathcal{O}, \text { free }}-\gamma_{\mathcal{O}}
$$

where $\gamma_{\mathcal{O}}$ is the anomalous dimension of the operator [44]. Since $\gamma_{\mathcal{O}}$ arises from the gauge interaction, it can be expressed as the power series

$$
\gamma^{(\mathcal{O})}=\sum_{\ell=1}^{\infty} c_{\gamma, \ell}^{(\mathcal{O})} a^{\ell},
$$

where $c_{\gamma, \ell}^{\mathcal{O}}$ is the $\ell$-loop coefficient.

As stated in the introduction, we shall consider the gauge-invariant operators $\mathcal{O}_{\mu_{1} \ldots \mu_{j}}=\bar{\psi} \gamma_{\mu_{1}} D_{\mu_{2}} \ldots D_{\mu_{j}} \psi$ and $\mathcal{O}_{\lambda \mu_{1} \ldots \mu_{j}}=\bar{\psi} \sigma_{\lambda \mu_{1}} D_{\mu_{2}} \ldots D_{\mu_{j}} \psi$, where

$$
\sigma_{\lambda \mu_{1}}=\frac{i}{2}\left[\gamma_{\lambda}, \gamma_{\mu_{1}}\right]
$$

We focus on the operators with $1 \leq j \leq 3$. We introduce the following compact notation for these operators:

$$
\begin{gathered}
\mathcal{O}_{\mu_{1} \mu_{2}}^{(\gamma D)} \equiv \bar{\psi} \gamma_{\mu_{1}} D_{\mu_{2}} \psi, \\
\mathcal{O}_{\mu_{1} \mu_{2} \mu_{3}}^{(\gamma D D)} \equiv \bar{\psi} \gamma_{\mu_{1}} D_{\mu_{2}} D_{\mu_{3}} \psi, \\
\mathcal{O}_{\mu_{1} \mu_{2} \mu_{3} \mu_{4}}^{(\gamma D D D)} \equiv \bar{\psi} \gamma_{\mu_{1}} D_{\mu_{2}} D_{\mu_{3}} D_{\mu_{4}} \psi, \\
\mathcal{O}_{\lambda \mu_{1} \mu_{2}}^{(\sigma D)} \equiv \bar{\psi} \sigma_{\lambda \mu_{1}} D_{\mu_{2}} \psi, \\
\mathcal{O}_{\lambda \mu_{1} \mu_{2} \mu_{3}}^{(\sigma D D)} \equiv \bar{\psi} \sigma_{\lambda \mu_{1}} D_{\mu_{2}} D_{\mu_{3}} \psi,
\end{gathered}
$$

and

$$
\mathcal{O}_{\lambda \mu_{1} \mu_{2} \mu_{3}}^{(\sigma D D)} \equiv \bar{\psi} \sigma_{\lambda \mu_{1}} D_{\mu_{2}} D_{\mu_{3}} D_{\mu_{4}} \psi
$$

For brevity of notation, we suppress the flavor indices on the fields $\psi$.

For a given operator $\mathcal{O}$, we write the schemeindependent expansion of its anomalous dimension $\gamma^{(\mathcal{O})}$ evaluated at the IRFP, denoted $\gamma_{\mathrm{IR}}^{(\mathcal{O})}$, as 


$$
\gamma_{\mathrm{IR}}^{(\mathcal{O})}=\sum_{n=1}^{\infty} \kappa_{n}^{(\mathcal{O})} \Delta_{f}{ }^{n}
$$

The truncation of right-hand side of Eq. (2.10) to maximal power $p$ is denoted

$$
\gamma_{\mathrm{IR}, \Delta_{f}^{p}}^{(\mathcal{O})}=\sum_{n=1}^{p} \kappa_{n}^{(\mathcal{O})} \Delta_{f}{ }^{n} .
$$

We use a further shorthand notation for the anomalous dimensions in which the superscript in $\gamma_{\mathrm{IR}}^{(\mathcal{O})}$ is replaced by a symbol for the quantity standing between $\bar{\psi}$ and $\psi$ in the operator $\mathcal{O}$. These shorthand symbols are as follows: $\gamma_{\mathrm{IR}}^{(\gamma D)}$ for the anomalous dimension of the operator $\mathcal{O}_{\mu_{1} \mu_{2}}^{(\gamma D)}=$ $\bar{\psi} \gamma_{\mu_{1}} D_{\mu_{2}} \psi$ at the IRFP, and so forth for the other operators. In comparing with our previous calculations in [34-39], we also use the notation $\gamma_{\mathrm{IR}}^{(1)}$ and $\gamma_{\mathrm{IR}}^{(\sigma)}$ for the anomalous dimensions of $\bar{\psi} \psi$ and $\bar{\psi} \sigma_{\lambda \mu_{1}} \psi$ at the IRFP. (The anomalous dimension $\gamma_{\mathrm{IR}}^{(\sigma)}$ was denoted $\gamma_{T, \mathrm{IR}}$ in [36], where the subscript $T$ referred to the Dirac tensor $\sigma_{\mu \nu}$.)

As discussed in $[34,36]$, the calculation of the coefficient $\kappa_{n}^{(\mathcal{O})}$ in Eq. (2.10) requires, as inputs, the beta function coefficients at loop order $1 \leq \ell \leq n+1$ and the anomalous dimension coefficients $c_{\gamma, \ell}^{(\mathcal{O})}$ at loop order $1 \leq \ell \leq n$. The method of calculation requires that the IR fixed point must be exact, which is the case in the non-Abelian Coulomb phase. As in our earlier work [34-39], we thus restrict our consideration to the non-Abelian Coulomb phase (conformal window) [45]. For a given gauge group $G$ and fermion representation $R$, the conformal window extends from an upper end at $N_{f}=N_{u}$ to a lower end at a value that is commonly denoted $N_{f, c r}$. In contrast to the exactly known value of $N_{u}$ [given in Eq. (1.6)], the value of $N_{f, c r}$ is not precisely known and has been investigated extensively for several groups $G$ and fermion representations $R$ [41,42,45]. For values of $N_{f}$ in the non-Abelian Coulomb phase such that $\Delta_{f}$ is not too large, one may expect the expansion (2.10) of $\gamma_{\mathrm{IR}}^{(\mathcal{O})}$ in a series in powers of $\Delta_{f}$ to yield reasonably accurate perturbative calculations of the anomalous dimension. In our earlier works, using our explicit calculations, we have shown that this is, in fact, the case.

We recall some relevant properties of the theory regarding global flavor symmetries. Because the $N_{f}$ fermions are massless, the Lagrangian is invariant under the classical global flavor $(f l)$ symmetry $G_{f l, c l}=\mathrm{U}\left(N_{f}\right)_{L} \otimes \mathrm{U}\left(N_{f}\right)_{R}$, or equivalently,

$G_{f l, c l}=\mathrm{SU}\left(N_{f}\right)_{L} \otimes \mathrm{SU}\left(N_{f}\right)_{R} \otimes \mathrm{U}(1)_{V} \otimes \mathrm{U}(1)_{A}$

(where $V$ and $A$ denote vector and axial-vector). The $\mathrm{U}(1)_{V}$ represents fermion number, which is conserved by the bilinear operators that we consider. The $\mathrm{U}(1)_{A}$ symmetry is broken by instantons, so the actual nonanomalous global flavor symmetry is

$$
G_{f l}=\mathrm{SU}\left(N_{f}\right)_{L} \otimes \mathrm{SU}\left(N_{f}\right)_{R} \otimes \mathrm{U}(1)_{V} .
$$

This $G_{f l}$ symmetry is respected in the non-Abelian Coulomb phase, since there is no spontaneous chiral symmetry breaking in this phase [41,42]. For our operators, the flavor matrix between $\bar{\psi}$ and $\psi$ is either the identity or the operator $T_{a}$, a generator of $\mathrm{SU}\left(N_{f}\right)$, which can be viewed as acting either to the right on $\psi$ or to the left on $\bar{\psi}$. These yield the same anomalous dimensions [46]. As a consequence of the unbroken global flavor symmetry, our operators transform as representations of the global flavor group $G_{f l}$. The invariance under the full $G_{f l}$ in the nonAbelian Coulomb phase is different from the situation in the QCD-like phase at smaller $N_{f}$, where the chiral part of $G_{f l}$ is spontaneously broken by the QCD bilinear quark condensate to the vectorial subgroup $\mathrm{SU}\left(N_{f}\right)_{V}$ and operators are classified according to whether they are singlet or nonsinglet (adjoint) under this vectorial $\mathrm{SU}\left(N_{f}\right)$ symmetry. In particular, in the consideration of flavor-singlet operators, in QCD, one must take into account mixing with gluonic operators. Here, in contrast, there is no mixing between any of our bilinear fermion operators and gluonic operators, since the latter are singlets under $G_{f l}$.

The operators $\mathcal{O}$ with an even number of Dirac $\gamma$ matrices, symbolically denoted $\Gamma_{e}$, link left with right chiral components of $\psi$, while the operators $\mathcal{O}$ with an odd number of Dirac $\gamma$ matrices, $\Gamma_{o}$, link left with left and right with right components:

$$
\begin{aligned}
& \bar{\psi} \Gamma_{e} \psi=\bar{\psi}_{L} \Gamma_{e} \psi_{R}+\bar{\psi}_{R} \Gamma_{e} \psi_{L}, \\
& \bar{\psi} \Gamma_{o} \psi=\bar{\psi}_{L} \Gamma_{o} \psi_{L}+\bar{\psi}_{R} \Gamma_{o} \psi_{R},
\end{aligned}
$$

where $\bar{\psi}=\psi^{\dagger} \gamma_{0}$. In the non-Abelian Coulomb phase where the flavor symmetry is (2.13), one may regard the $T_{b}$ in the term $\bar{\psi}_{L} T_{b} \psi_{R}$ acting to the right as an element of $\operatorname{SU}\left(N_{f}\right)_{R}$ and acting to the left as an element of $\mathrm{SU}\left(N_{f}\right)_{L}$.

Given that the theory at the IR fixed point is conformally invariant [16], there is an important lower bound on the full dimension of an operator $\mathcal{O}$ and hence, with our definition (2.1), an upper bound on the anomalous dimension $\gamma^{(\mathcal{O})}$ that follows from the conformal invariance. To state this, we first recall that a (finite-dimensional) representation of the Lorentz group is specified by the set $\left(j_{1}, j_{2}\right)$, where $j_{1}$ and $j_{2}$ take on non-negative integral or half-integral values [47]. A Lorentz scalar operator thus transforms as $(0,0)$, a Lorentz vector as $(1 / 2,1 / 2)$, an antisymmetric tensor like the field-strength tensor $F_{\mu \nu}^{a}$ as $(1,0) \oplus(0,1)$, etc. Then the requirement of unitarity in a conformally invariant theory implies the lower bound [48] 


$$
D_{\mathcal{O}} \geq j_{1}+j_{2}+1
$$

i.e., the upper bound

$$
\gamma_{\mathcal{O}} \leq D_{\mathcal{O}, \text { free }}-\left(j_{1}+j_{2}+1\right) .
$$

We have studied the constraints from the upper bound (2.17) in our previous calculations of anomalous dimensions in [22,25,36-39]. Anticipating the results given below, since our calculations yield negative values for the anomalous dimensions of higher-spin Wilson operators, they obviously satisfy these conformality upper bounds.

\section{SOME GENERAL STRUCTURAL PROPERTIES OF $\gamma_{\mathrm{IR}}^{(\mathcal{O})}$}

From our previous calculations [34-39] for the anomalous dimensions of $\bar{\psi} \psi$ and $\bar{\psi} \sigma_{\mu \nu} \psi$, in conjunction with our new results on the anomalous dimensions $\gamma_{\mathrm{IR}}^{(\mathcal{O})}$ of higherspin twist-2 bilinear fermion operators $\mathcal{O}$, we find some general structural properties of the coefficients $\kappa_{n}^{(\mathcal{O})}$ in the scheme-independent series expansions of the anomalous dimensions $\gamma_{\mathrm{IR}}^{(\mathcal{O})}$. These involve various group invariants, including the quadratic Casimir invariants $C_{A} \equiv C_{2}(G)$, $C_{f} \equiv C_{2}(R)$, the trace invariant $T(R)$, and the quartic trace invariants $d_{R}^{a b c d} d_{R^{\prime}}^{a b c d} / d_{A}$, where $d_{A}$ denotes the dimension of the adjoint representation $[18,49]$. For compact notation, it is convenient to define a factor that occurs in the denominators of these $\kappa_{n}^{(\mathcal{O})}$ coefficients, namely.

$$
D=7 C_{A}+11 C_{f}
$$

(not to be confused with covariant derivative). We exhibit this general form here, using $a_{j, k}^{(\mathcal{O})}$ for various (constant) numerical coefficients:

$$
\begin{gathered}
\kappa_{1}^{(\mathcal{O})}=c_{1}^{(\mathcal{O})} \frac{C_{f} T_{f}}{C_{A} D}, \\
\kappa_{2}^{(\mathcal{O})}=\frac{C_{f} T_{f}^{2}\left(a_{2,1}^{(\mathcal{O})} C_{A}^{2}+a_{2,2}^{(\mathcal{O})} C_{A} C_{f}+a_{2,3}^{(\mathcal{O})} C_{f}^{2}\right)}{C_{A}^{2} D^{3}},
\end{gathered}
$$

and

$$
\begin{aligned}
\kappa_{3}^{(\mathcal{O})}= & \frac{C_{f} T_{f}}{C_{A}^{4} D^{5}}\left[a_{3,1}^{(\mathcal{O})} C_{A}^{5} T_{f}^{2}+a_{3,2}^{(\mathcal{O})} C_{A}^{4} C_{f} T_{f}^{2}+a_{3,3}^{(\mathcal{O})} C_{A}^{3} C_{f}^{2} T_{f}^{2}+a_{3,4}^{(\mathcal{O})} C_{A}^{2} C_{f}^{3} T_{f}^{2}+a_{3,5}^{(\mathcal{O})} C_{A} C_{f}^{4} T_{f}\right. \\
& +a_{3,6}^{(\mathcal{O})} C_{A} T_{f}^{2} \frac{d_{A}^{a b c d} d_{A}^{a b c d}}{d_{A}}+a_{3,7}^{(\mathcal{O})} C_{f} T_{f}^{2} \frac{d_{A}^{a b c d} d_{A}^{a b c d}}{d_{A}}+a_{3,8}^{(\mathcal{O})} C_{A}^{2} T_{f} \frac{d_{R}^{a b c d} d_{A}^{a b c d}}{d_{A}}+a_{3,9}^{(\mathcal{O})} C_{A} C_{f} T_{f} \frac{d_{R}^{a b c d} d_{A}^{a b c d}}{d_{A}} \\
& \left.+a_{3,10}^{(\mathcal{O})} C_{A}^{3} \frac{d_{R}^{a b c d} d_{R}^{a b c d}}{d_{A}}+a_{3,11}^{(\mathcal{O})} C_{A}^{2} C_{f} \frac{d_{R}^{a b c d} d_{R}^{a b c d}}{d_{A}}\right] .
\end{aligned}
$$

\section{ANOMALOUS DIMENSIONS $\gamma_{\mathrm{IR}}^{(\mathcal{O})}$ OF HIGHER-SPIN OPERATORS}

\section{A. General}

In this section we present the results of our calculations of the coefficients in the scheme-independent series expansions up to $O\left(\Delta_{f}^{3}\right)$ for the various higher-spin operators considered here. As was noted above, the calculation of the $O\left(\Delta_{f}^{n}\right)$ coefficient, $\kappa_{n}^{(\mathcal{O})}$, for the anomalous dimension of an operator $\mathcal{O}$ at the IRFP requires, as inputs, the beta function coefficients at loop order $1 \leq \ell \leq n+1$ and the anomalous dimension coefficients $c_{\ell}^{(\mathcal{O})}$ at loop order $1 \leq \ell \leq n$. Hence, we use the beta function coefficients from one-loop up to the four-loop level $[10,19,50,51]$, together with the anomalous dimension coefficients calculated in the conventional series expansion in powers of $a$ up to the three-loop level $[11,46,52-57]$. The higher-order terms in the beta function and anomalous dimensions that we use have been calculated in the $\overline{\mathrm{MS}}$ scheme [58], but our results are independent of this since they are scheme-independent. (The beta function has actually been calculated up to five-loop order $[59,60]$, but these results will not be needed here.)

$$
\text { B. } \gamma_{\mathrm{IR}}^{(\gamma D)}
$$

For the anomalous dimension $\gamma_{\mathrm{IR}}^{(\gamma D)}$ of the operator $\bar{\psi} \gamma_{\mu_{1}} D_{\mu_{2}} \psi$ at the IRFP, we calculate

$$
\begin{gathered}
\kappa_{1}^{(\gamma D)}=-\frac{2^{6} C_{f} T_{f}}{3^{2} C_{A} D}, \\
\kappa_{2}^{(\gamma D)}=\frac{2^{5} C_{f} T_{f}^{2}\left(693 C_{A}^{2}-3104 C_{A} C_{f}-1540 C_{f}^{2}\right)}{3^{5} C_{A}^{2} D^{3}},
\end{gathered}
$$

and 


$$
\begin{aligned}
\kappa_{3}^{(\gamma D)}= & -\frac{2^{5} C_{f} T_{f}}{3^{8} C_{A}^{4} D^{5}}\left[C_{A}^{5} T_{f}^{2}\left(-202419+1016064 \zeta_{3}\right)+C_{A}^{4} C_{f} T_{f}^{2}\left(2764440+145152 \zeta_{3}\right)\right. \\
& +C_{A}^{3} C_{f}^{2} T_{f}^{2}\left(8940028-5038848 \zeta_{3}\right)+C_{A}^{2} C_{f}^{3} T_{f}^{2}\left(-7341488-1140480 \zeta_{3}\right)+C_{A} C_{f}^{4} T_{f}^{2}\left(3841024+5018112 \zeta_{3}\right) \\
& +C_{A} T_{f}^{2} \frac{d_{A}^{a b c d} d_{A}^{a b c d}}{d_{A}}\left(-161280+4257792 \zeta_{3}\right)+C_{f} T_{f}^{2} \frac{d_{A}^{a b c d} d_{A}^{a b c d}}{d_{A}}\left(-253440+6690816 \zeta_{3}\right) \\
& +C_{A}^{2} T_{f} \frac{d_{R}^{a b c d} d_{A}^{a b c d}}{d_{A}}\left(2838528-27675648 \zeta_{3}\right)+C_{A} C_{f} T_{f} \frac{d_{R}^{a b c d} d_{A}^{a b c d}}{d_{A}}\left(4460544-43490304 \zeta_{3}\right) \\
& \left.+C_{A}^{3} \frac{d_{R}^{a b c d} d_{R}^{a b c d}}{d_{A}}\left(-10733184+23417856 \zeta_{3}\right)+C_{A}^{2} C_{f} \frac{d_{R}^{a b c d} d_{R}^{a b c d}}{d_{A}}\left(-16866432+36799488 \zeta_{3}\right)\right] .
\end{aligned}
$$

In these expressions and the following ones, we have indicated the factorizations of the numbers in the denominators, since they are rather simple. In general, the numbers in the numerators do not have such simple factorizations.

With these coefficients, the anomalous dimension $\gamma_{\mathrm{IR}}^{(\gamma D)}$ calculated to order $O\left(\Delta_{f}^{p}\right)$, denoted $\gamma_{\mathrm{IR}, F, \Delta_{f}^{p}}^{(\gamma D)}$, is given by Eq. (2.11) with $\mathcal{O}=\bar{\psi} \gamma_{\mu_{1}} D_{\mu_{2}} \psi$. Our results here yield $\gamma_{\mathrm{IR}, F, \Delta_{f}^{p}}^{(\gamma D)}$ with $p=1,2,3$. Analogous statements apply to the anomalous dimensions of the other operators for which we have performed calculations, and we proceed to present the coefficients for these next.

\section{C. $\gamma_{\mathbf{I R}}^{(\gamma D D)}$}

For the anomalous dimension $\gamma_{\mathrm{IR}}^{(\gamma D D)}$ of the operator $\bar{\psi} \gamma_{\mu_{1}} D_{\mu_{2}} D_{\mu_{3}} \psi$ at the IRFP, we calculate

$$
\kappa_{1}^{(\gamma D D)}=-\frac{100 C_{f} T_{f}}{3^{2} C_{A} D}
$$

$$
\kappa_{2}^{(\gamma D D)}=\frac{10 C_{f} T_{f}^{2}\left(5103 C_{A}^{2}-14017 C_{A} C_{f}-9383 C_{f}^{2}\right)}{3^{5} C_{A}^{2} D^{3}},
$$

and

$$
\begin{aligned}
\kappa_{3}^{(\gamma D D)}= & -\frac{10 C_{f} T_{f}}{3^{8} C_{A}^{4} D^{5}}\left[C_{A}^{5} T_{f}^{2}\left(1538649+2794176 \zeta_{3}\right)+C_{A}^{4} C_{f} T_{f}^{2}\left(14860881+399168 \zeta_{3}\right)\right. \\
& +C_{A}^{3} C_{f}^{2} T_{f}^{2}\left(40821518-13856832 \zeta_{3}\right)+C_{A}^{2} C_{f}^{3} T_{f}^{2}\left(-35403412-3136320 \zeta_{3}\right)+C_{A} C_{f}^{4} T_{f}^{2}\left(19308575+13799808 \zeta_{3}\right) \\
& +C_{A} T_{f}^{2} \frac{d_{A}^{a b c d} d_{A}^{a b c d}}{d_{A}}\left(-806400+21288960 \zeta_{3}\right)+C_{f} T_{f}^{2} \frac{d_{A}^{a b c d} d_{A}^{a b c d}}{d_{A}}\left(-1267200+33454080 \zeta_{3}\right) \\
& +C_{A}^{2} T_{f} \frac{d_{R}^{a b c d} d_{A}^{a b c d}}{d_{A}}\left(14192640-138378240 \zeta_{3}\right)+C_{A} C_{f} T_{f} \frac{d_{R}^{a b c d} d_{A}^{a b c d}}{d_{A}}\left(22302720-217451520 \zeta_{3}\right) \\
& \left.+C_{A}^{3} \frac{d_{R}^{a b c d} d_{R}^{a b c d}}{d_{A}}\left(-53665920+117089280 \zeta_{3}\right)+C_{A}^{2} C_{f} \frac{d_{R}^{a b c d} d_{R}^{a b c d}}{d_{A}}\left(-84332160+183997440 \zeta_{3}\right)\right]
\end{aligned}
$$

\section{D. $\gamma_{\mathrm{IR}}^{(\gamma D D D)}$}

Proceeding to the anomalous dimension $\gamma_{\mathrm{IR}}^{(\gamma D D D)}$ of the operator $\bar{\psi} \gamma_{\mu_{1}} D_{\mu_{2}} D_{\mu_{3}} D_{\mu_{4}} \psi$ at the IRFP, we find

$$
\begin{gathered}
\kappa_{1}^{(\gamma D D D)}=-\frac{628 C_{f} T_{f}}{3^{2} \cdot 5 C_{A} D}, \\
\kappa_{2}^{(\gamma D D D)}=\frac{2 C_{f} T_{f}^{2}\left(4550175 C_{A}^{2}-10373329 C_{A} C_{f}-7719767 C_{f}^{2}\right)}{3^{5} \cdot 5^{3} C_{A}^{2} D^{3}},
\end{gathered}
$$


and

$$
\begin{aligned}
\kappa_{3}^{(\gamma D D D)}= & \frac{2 C_{f} T_{f}}{3^{8} \cdot 5^{5} C_{A}^{4} D^{5}}\left[C_{A}^{5} T_{f}^{2}\left(-67181774625-45691128000 \zeta_{3}\right)+C_{A}^{4} C_{f} T_{f}^{2}\left(-318706112025-6527304000 \zeta_{3}\right)\right. \\
& +C_{A}^{3} C_{f}^{2} T_{f}^{2}\left(-720947009518+226590696000 \zeta_{3}\right)+C_{A}^{2} C_{f}^{3} T_{f}^{2}\left(709569531572+51285960000 \zeta_{3}\right) \\
& +C_{A} C_{f}^{4} T_{f}^{2}\left(-433168554247-225658224000 \zeta_{3}\right) \\
& +C_{A} T_{f}^{2} \frac{d_{A}^{a b c d} d_{A}^{a b c d}}{d_{A}}\left(15825600000-417795840000 \zeta_{3}\right)+C_{f} T_{f}^{2} \frac{d_{A}^{a b c d} d_{A}^{a b c d}}{d_{A}}\left(24868800000-6565363200000 \zeta_{3}\right) \\
& +C_{A}^{2} T_{f} \frac{d_{R}^{a b c d} d_{A}^{a b c d}}{d_{A}}\left(-278530560000+2715672960000 \zeta_{3}\right) \\
& +C_{A} C_{f} T_{f} \frac{d_{R}^{a b c d} d_{A}^{a b c d}}{d_{A}}\left(-437690880000+4267486080000 \zeta_{3}\right) \\
& +C_{A}^{3} \frac{d_{R}^{a b c d} d_{R}^{a b c d}}{d_{A}}\left(1053193680000-2297877120000 \zeta_{3}\right) \\
& \left.+C_{A}^{2} C_{f} \frac{d_{R}^{a b c d} d_{R}^{a b c d}}{d_{A}}\left(1655018640000-3610949760000 \zeta_{3}\right)\right] \\
& \text { E. } \gamma_{\mathbf{I R}}^{(\sigma D)}
\end{aligned}
$$

For the anomalous dimension $\gamma_{\mathrm{IR}}^{(\sigma D)}$ of the operator $\bar{\psi} \sigma_{\lambda \mu_{1}} D_{\mu_{2}} \psi$ at the IRFP, we calculate

$$
\begin{aligned}
& \kappa_{1}^{(\sigma D)}=-\frac{8 C_{f} T_{f}}{C_{A} D} \\
& \kappa_{2}^{(\sigma D)}=\frac{4 C_{f} T_{f}^{2}\left(77 C_{A}^{2}-348 C_{A} C_{f}-176 C_{f}^{2}\right)}{3 C_{A}^{2} D^{3}}, \\
& \kappa_{3}^{(\sigma D)}=\frac{4 C_{f} T_{f}}{3^{4} C_{A}^{4} D^{5}}\left[13083 C_{A}^{5} T_{f}^{2}-240492 C_{A}^{4} C_{f} T_{f}^{2}-819408 C_{A}^{3} C_{f}^{2} T_{f}^{2}+738144 C_{A}^{2} C_{f}^{3} T_{f}^{2}-662112 C_{A} C_{f}^{4} T_{f}^{2}\right. \\
& +C_{A} T_{f}^{2} \frac{d_{A}^{a b c d} d_{A}^{a b c d}}{d_{A}}\left(17920-473088 \zeta_{3}\right)+C_{f} T_{f}^{2} \frac{d_{A}^{a b c d} d_{A}^{a b c d}}{d_{A}}\left(28160-743424 \zeta_{3}\right) \\
& +C_{A}^{2} T_{f} \frac{d_{R}^{a b c d} d_{A}^{a b c d}}{d_{A}}\left(-315392+3075072 \zeta_{3}\right)+C_{A} C_{f} T_{f} \frac{d_{R}^{a b c d} d_{A}^{a b c d}}{d_{A}}\left(-495616+4832256 \zeta_{3}\right) \\
& \left.+C_{A}^{3} \frac{d_{R}^{a b c d} d_{R}^{a b c d}}{d_{A}}\left(1192576-2601984 \zeta_{3}\right)+C_{A}^{2} C_{f} \frac{d_{R}^{a b c d} d_{R}^{a b c d}}{d_{A}}\left(1874048-4088832 \zeta_{3}\right)\right] . \\
& \text { F. } \gamma_{\text {IR }}^{(\sigma D D)}
\end{aligned}
$$

For the anomalous dimension $\gamma_{\mathrm{IR}}^{(\sigma D D)}$ we calculate

$$
\begin{gathered}
\kappa_{1}^{(\sigma D D)}=-\frac{104 C_{f} T_{f}}{3^{2} C_{A} D}, \\
\kappa_{2}^{(\sigma D D)}=\frac{4 C_{f} T_{f}^{2}\left(12537 C_{A}^{2}-36292 C_{A} C_{f}-22352 C_{f}^{2}\right)}{3^{5} C_{A}^{2} D^{3}},
\end{gathered}
$$


and

$$
\begin{aligned}
\kappa_{3}^{(\sigma D D)}= & -\frac{2^{2} C_{f} T_{f}}{3^{8} C_{A}^{4} D^{5}}\left[C_{A}^{5} T_{f}^{2}\left(2935737+4064256 \zeta_{3}\right)+C_{A}^{4} C_{f} T_{f}^{2}\left(39906468+580608 \zeta_{3}\right)\right. \\
& +C_{A}^{3} C_{f}^{2} T_{f}^{2}\left(107242456-20155392 \zeta_{3}\right)+C_{A}^{2} C_{f}^{3} T_{f}^{2}\left(-102128048-4561920 \zeta_{3}\right) \\
& +C_{A} C_{f}^{4} T_{f}^{2}\left(43045024+20072448 \zeta_{3}\right) \\
& +3 C_{A} T_{f}^{2} \frac{d_{A}^{a b c d} d_{A}^{a b c d}}{d_{A}}\left(-698880+18450432 \zeta_{3}\right)+3 C_{f} T_{f}^{2} \frac{d_{A}^{a b c d} d_{A}^{a b c d}}{d_{A}}\left(-1098240+28993536 \zeta_{3}\right) \\
& +3 C_{A}^{2} T_{f} \frac{d_{R}^{a b c d} d_{A}^{a b c d}}{d_{A}}\left(12300288-119927808 \zeta_{3}\right)+3 C_{A} C_{f} T_{f} \frac{d_{R}^{a b c d} d_{A}^{a b c d}}{d_{A}}\left(19329024-188457984 \zeta_{3}\right) \\
& \left.+3 C_{A}^{3} \frac{d_{R}^{a b c d} d_{R}^{a b c d}}{d_{A}}\left(-46510464+101477376 \zeta_{3}\right)+3 C_{A}^{2} C_{f} \frac{d_{R}^{a b c d} d_{R}^{a b c d}}{d_{A}}\left(-73087872+159464448 \zeta_{3}\right)\right] . \\
& \text { G. } \gamma_{\mathbf{I R}}^{(\sigma D D D)}
\end{aligned}
$$

Finally, for the anomalous dimension $\gamma_{\mathrm{IR}}^{\sigma D D D)}$ we obtain

$$
\begin{gathered}
\kappa_{1}^{(\sigma D D D)}=-\frac{2^{7} C_{f} T_{f}}{3^{2} C_{A} D}, \\
\kappa_{2}^{(\sigma D D D)}=\frac{2^{3} C_{f} T_{f}^{2}\left(9219 C_{A}^{2}-21185 C_{A} C_{f}-15664 C_{f}^{2}\right)}{3^{5} C_{A}^{2} D^{3}},
\end{gathered}
$$

and

$$
\begin{aligned}
\kappa_{3}^{(\sigma D D D)}= & -\frac{2^{3} C_{f} T_{f}}{3^{8} C_{A}^{4} D^{5}}\left[C_{A}^{5} T_{f}^{2}\left(5213502+2667168 \zeta_{3}\right)+C_{A}^{4} C_{f} T_{f}^{2}\left(25185069+381024 \zeta_{3}\right)\right. \\
& +C_{A}^{3} C_{f}^{2} T_{f}^{2}\left(58268711-13226976 \zeta_{3}\right)+C_{A}^{2} C_{f}^{3} T_{f}^{2}\left(-56962840-2993760 \zeta_{3}\right) \\
& +C_{A} C_{f}^{4} T_{f}^{2}\left(36476660+13172544 \zeta_{3}\right) \\
& +C_{A} T_{f}^{2} \frac{d_{A}^{a b c d} d_{A}^{a b c d}}{d_{A}}\left(-1290240+34062336 \zeta_{3}\right)+C_{f} T_{f}^{2} \frac{d_{A}^{a b c d} d_{A}^{a b c d}}{d_{A}}\left(-2027520+53526528 \zeta_{3}\right) \\
& +C_{A}^{2} T_{f} \frac{d_{R}^{a b c d} d_{A}^{a b c d}}{d_{A}}\left(22708224-221405184 \zeta_{3}\right)+C_{A} C_{f} T_{f} \frac{d_{R}^{a b c d} d_{A}^{a b c d}}{d_{A}}\left(35684352-347922432 \zeta_{3}\right) \\
& \left.+C_{A}^{3} \frac{d_{R}^{a b c d} d_{R}^{a b c d}}{d_{A}}\left(-85865472+187342848 \zeta_{3}\right)+C_{A}^{2} C_{f} \frac{d_{R}^{a b c d} d_{R}^{a b c d}}{d_{A}}\left(-134931456+294395904 \zeta_{3}\right)\right] .
\end{aligned}
$$

\section{EVALUATION OF $\kappa_{n}^{(\mathcal{O})}$ FOR $G=\mathrm{SU}\left(N_{c}\right)$ AND $R=F$}

In this section we evaluate our general results for these anomalous dimensions $\gamma_{\mathrm{IR}}^{(\mathcal{O})}$ in the important special case where the gauge group is $G=\mathrm{SU}\left(N_{c}\right)$ and the $N_{f}$ fermions are in the fundamental representation of this group, $R=F$.

$$
\text { A. } \gamma_{\mathbf{I R}, \mathbf{S U}\left(N_{c}\right), \boldsymbol{F}}^{(\gamma \boldsymbol{D})}
$$$$
\kappa_{2, \mathrm{SU}\left(N_{c}\right), F}^{(\gamma D)}=-\frac{2^{5}\left(N_{c}^{2}-1\right)\left(1244 N_{c}^{4}-2322 N_{c}^{2}+385\right)}{3^{5} N_{c}^{2}\left(25 N_{c}^{2}-11\right)^{3}},
$$

$$
\kappa_{1, \mathrm{SU}\left(N_{c}\right), F}^{(\gamma D)}=-\frac{2^{5}\left(N_{c}^{2}-1\right)}{3^{2} N_{c}\left(25 N_{c}^{2}-11\right)}
$$

Substituting $G=\mathrm{SU}\left(N_{c}\right)$ and $R=F$ in our general results (4.1)-(4.3), we obtain 


$$
\begin{aligned}
\kappa_{3, \mathrm{SU}\left(N_{c}\right), F}^{(\gamma D)}= & -\frac{2^{6}\left(N_{c}^{2}-1\right)}{3^{8} N_{c}^{3}\left(25 N_{c}^{2}-11\right)^{5}}\left[2137786 N_{c}^{8}+\left(1831104-9784800 \zeta_{3}\right) N_{c}^{6}\right. \\
& \left.+\left(-15928259+36575712 \zeta_{3}\right) N_{c}^{4}+\left(6282342-14911776 \zeta_{3}\right) N_{c}^{2}+240064+313632 \zeta_{3}\right] .
\end{aligned}
$$

Then, for this case $G=\mathrm{SU}(3), R=F$, the anomalous dimension $\gamma_{\mathrm{IR}}^{(\gamma D)}$ calculated to order $O\left(\Delta_{f}^{p}\right)$, denoted $\gamma_{\mathrm{IR}, F, \Delta_{f}^{p}}^{(\gamma D)}$, is given by Eq. (2.11) with $\mathcal{O}=\bar{\psi} \gamma_{\mu_{1}} D_{\mu_{2}} \psi$.

$$
\text { B. } \gamma_{\mathbf{I R}, \mathbf{S U}\left(N_{c}\right), \boldsymbol{F}}^{(\gamma D D)}
$$

Substituting $G=\mathrm{SU}\left(N_{c}\right)$ and $R=F$ in our general results (4.4)-(4.6), we obtain

$$
\begin{gathered}
\kappa_{1, \mathrm{SU}\left(N_{c}\right), F}^{(\gamma D D)}=-\frac{50\left(N_{c}^{2}-1\right)}{3^{2} N_{c}\left(25 N_{c}^{2}-11\right)}, \\
\kappa_{2, \mathrm{SU}\left(N_{c}\right), F}^{(\gamma D D)}=-\frac{5\left(N_{c}^{2}-1\right)\left(17005 N_{c}^{4}-46800 N_{c}^{2}+9383\right)}{2 \cdot 3^{5} N_{c}^{2}\left(25 N_{c}^{2}-11\right)^{3}},
\end{gathered}
$$

and

$$
\begin{aligned}
\kappa_{3, \mathrm{SU}\left(N_{c}\right), F}^{(\gamma D D)}= & -\frac{5\left(N_{c}^{2}-1\right)}{2^{2} \cdot 3^{8} N_{c}^{3}\left(25 N_{c}^{2}-11\right)^{5}}\left[207341255 N_{c}^{8}+\left(160969860-841104000 \zeta_{3}\right) N_{c}^{6}\right. \\
& \left.+\left(-1281330310+2919058560 \zeta_{3}\right) N_{c}^{4}+\left(499565484-1152911232 \zeta_{3}\right) N_{c}^{2}+19308575+13799808 \zeta_{3}\right] .
\end{aligned}
$$

$$
\text { C. } \gamma_{\operatorname{IR}, \mathbf{S U}\left(\boldsymbol{N}_{\boldsymbol{c}}\right), \boldsymbol{F}}^{(\gamma D D D)}
$$

In a similar manner, from our general formulas (4.7)-(4.9), we find

$$
\begin{gathered}
\kappa_{1, \mathrm{SU}\left(N_{c}\right), F}^{(\gamma D D)}=-\frac{314\left(N_{c}^{2}-1\right)}{3^{2} \cdot 5 N_{c}\left(25 N_{c}^{2}-11\right)}, \\
\kappa_{2, \mathrm{SU}\left(N_{c}\right), F}^{(\gamma D D)}=-\frac{\left(N_{c}^{2}-1\right)\left(10265725 N_{c}^{4}-36186192 N_{c}^{2}+7719767\right)}{2 \cdot 3^{5} \cdot 5^{3} N_{c}^{2}\left(25 N_{c}^{2}-11\right)^{3}},
\end{gathered}
$$

and

$$
\begin{aligned}
\kappa_{3, \mathrm{SU}\left(N_{c}\right), F}^{(\gamma D D D)}= & -\frac{\left(N_{c}^{2}-1\right)}{2^{2} \cdot 3^{8} \cdot 5^{5} N_{c}^{3}\left(25 N_{c}^{2}-11\right)^{5}}\left[4581316819375 N_{c}^{8}+\left(3455659520100-16739946000000 \zeta_{3}\right) N_{c}^{6}\right. \\
& +\left(-25230047265878+57258530640000 \zeta_{3}\right) N_{c}^{4}+\left(9616576686156-22465759536000 \zeta_{3}\right) N_{c}^{2} \\
& \left.\left.+433168554247+225658224000 \zeta_{3}\right)\right] . \\
& \text { D. } \gamma_{\mathbf{I R}, \mathbf{S U}\left(N_{c}\right), \boldsymbol{F}}^{(\sigma \boldsymbol{D D})}
\end{aligned}
$$

From our general results (4.10)-(4.12), we obtain

$$
\begin{gathered}
\kappa_{1, \mathrm{SU}\left(N_{c}\right), F}^{(\sigma D)}=-\frac{4\left(N_{c}^{2}-1\right)}{N_{c}\left(25 N_{c}^{2}-11\right)}, \\
\kappa_{2, \mathrm{SU}\left(N_{c}\right), F}^{(\sigma D)}=-\frac{4\left(N_{c}^{2}-1\right)\left(141 N_{c}^{4}-262 N_{c}^{2}+44\right)}{3 N_{c}^{2}\left(25 N_{c}^{2}-11\right)^{3}},
\end{gathered}
$$


and

$$
\begin{aligned}
& \kappa_{3, \mathrm{SU}\left(N_{c}\right), F}^{(\sigma D)}=-\frac{2^{3}\left(N_{c}^{2}-1\right)}{3^{3} N_{c}^{3}\left(25 N_{c}^{2}-11\right)^{5}}\left[64843 N_{c}^{8}+\left(78610-422400 \zeta_{3}\right) N_{c}^{6}\right. \\
&\left.+\left(-565316+1347456 \zeta_{3}\right) N_{c}^{4}+\left(209836-511104 \zeta_{3}\right) N_{c}^{2}+13794\right] . \\
& \text { E. } \boldsymbol{\gamma}_{\mathbf{I R}, \mathbf{S U}\left(N_{c}\right), \boldsymbol{F}}^{(\sigma D D)}
\end{aligned}
$$

From our general results (4.13)-(4.15), we obtain

$$
\begin{gathered}
\kappa_{1, \mathrm{SU}\left(N_{c}\right), F}^{(\sigma D D)}=-\frac{52\left(N_{c}^{2}-1\right)}{3^{2} N_{c}\left(25 N_{c}^{2}-11\right)}, \\
\kappa_{2, \mathrm{SU}\left(N_{c}\right), F}^{(\sigma D D)}=-\frac{4\left(N_{c}^{2}-1\right)\left(11197 N_{c}^{4}-29322 N_{c}^{2}+5588\right)}{3^{5} N_{c}^{2}\left(25 N_{c}^{2}-11\right)^{3}},
\end{gathered}
$$

and

For this case we have

$$
\begin{aligned}
& \kappa_{3, \mathrm{SU}\left(N_{c}\right), F}^{(\sigma D D)}=-\frac{2^{3}\left(N_{c}^{2}-1\right)}{3^{8} N_{c}^{3}\left(25 N_{c}^{2}-11\right)^{5}}\left[31831693 N_{c}^{8}+\left(30539268-141782400 \zeta_{3}\right) N_{c}^{6}\right. \\
&+\left(-214403216+473734656 \zeta_{3}\right) N_{c}^{4}+\left(84228606-183845376 \zeta_{3}\right) N_{c}^{2} \\
&\left.+2690314+1254528 \zeta_{3}\right] . \\
& \text { F. } \boldsymbol{\gamma}_{\mathbf{I R}, \mathbf{S U}\left(N_{c}\right), \boldsymbol{F}}^{(\sigma D D D)}
\end{aligned}
$$

and

$$
\begin{aligned}
\kappa_{3, \mathrm{SU}\left(N_{c}\right), F}^{(\sigma D D D)}= & -\frac{2^{2}\left(N_{c}^{2}-1\right)}{3^{8} N_{c}^{3}\left(25 N_{c}^{2}-11\right)^{5}}\left[\left(90949802 N_{c}^{8}+\left(70557192-347943600 \zeta_{3}\right) N_{c}^{6}\right.\right. \\
& +\left(-511679503+1166243184 \zeta_{3}\right) N_{c}^{4}+\left(194401944-453269520 \zeta_{3}\right) N_{c}^{2} \\
& \left.+9119165+3293136 \zeta_{3}\right] .
\end{aligned}
$$

Below, where the meaning is clear, we will often omit the $\mathrm{SU}(3)$ in the subscript.

We remark on the signs of these coefficients. It is evident from Eqs. (4.1), (4.4), (4.7), (4.10), (4.13), and (4.16) that $\kappa_{1}^{(\gamma D)}, \kappa_{1}^{(\gamma D D)}, \kappa_{1}^{(\gamma D D D)}, \kappa_{1}^{(\sigma D)}, \kappa_{1}^{(\sigma D D)}$, and $\kappa_{1}^{(\sigma D D D)}$ are all negative for any $G$ and $R$. We find that the $O\left(\Delta_{f}^{2}\right)$ and $O\left(\Delta_{f}^{3}\right)$ coefficients, $\kappa_{2}^{(\mathcal{O})}$ and $\kappa_{3}^{(\mathcal{O})}$, for these operators are also negative for the theory with $G=\mathrm{SU}\left(N_{c}\right)$ and fermions in the fundamental representation, $R=F$, in the full range $N_{c} \geq 2$ of relevance here. In Table I we list the signs of these coefficients $\kappa_{n}^{(\mathcal{O})}$ for the operators in this theory. For comparison, we also include the signs of $\kappa_{n}^{(1)}$ for $\bar{\psi} \psi$ and $\kappa_{n}^{(\sigma)}$ for $\bar{\psi} \sigma_{\mu \nu} \psi$ that we obtained in our earlier calculations (which hold for all $N_{c}$ ).

It is interesting to note that for all of the higher-spin operators $\mathcal{O}$ that we consider, the anomalous dimensions $\gamma_{\text {IR }}^{(\mathcal{O})}$ that we calculate are negative (with our sign convention in (2.1) [44]). They thus have the same sign as the 
TABLE I. Signs of scheme-independent expansion coefficients $\kappa_{n}^{(\mathcal{O})}$ for gauge group $G=\mathrm{SU}\left(N_{c}\right)$ with $N_{c} \geq 2$ and fermion representation $R=F$ (fundamental).

\begin{tabular}{lccc}
\hline \hline $\mathcal{O}$ & $\kappa_{1, \mathrm{SU}\left(N_{c}\right), F}^{(\mathcal{O})}$ & $\kappa_{2, \mathrm{SU}\left(N_{c}\right), F}^{(\mathcal{O})}$ & $\kappa_{3, \mathrm{SU}\left(N_{c}\right), F}^{(\mathcal{O})}$ \\
\hline $\bar{\psi} \psi$ & + & + & + \\
$\bar{\psi} \sigma_{\lambda \mu_{1}} \psi$ & - & - & + \\
$\bar{\psi} \gamma_{\mu_{1}} D_{\mu_{2}} \psi$ & - & - & - \\
$\bar{\psi} \gamma_{\mu_{1}} D_{\mu_{2}} D_{\mu_{3}} \psi$ & - & - & - \\
$\bar{\psi} \gamma_{\mu_{1}} D_{\mu_{2}} D_{\mu_{3}} D_{\mu_{4}} \psi$ & - & - & - \\
$\bar{\psi} \sigma_{\lambda \mu_{1}} D_{\mu_{2}} \psi$ & - & - & - \\
$\bar{\psi} \sigma_{\lambda \mu_{1}} D_{\mu_{2}} D_{\mu_{3}} \psi$ & - & - & - \\
$\bar{\psi} \sigma_{\lambda \mu_{1}} D_{\mu_{2}} D_{\mu_{3}} D_{\mu_{4}} \psi$ & - & - & - \\
\hline \hline
\end{tabular}

TABLE II. Values of the anomalous dimension $\gamma_{\mathrm{IR}, F}^{(\gamma D)}$ calculated to $O\left(\Delta_{f}^{p}\right)$, denoted $\gamma_{\mathrm{IR}, F, \Delta_{f}^{p}}^{(\gamma D)}$, with $1 \leq p \leq 3$, for $G=\mathrm{SU}(3)$, as a function of $N_{f}$.

\begin{tabular}{llll}
\hline \hline$N_{f}$ & $\gamma_{\mathrm{IR}, F, \Delta_{f}}^{(\gamma D)}$ & $\gamma_{\mathrm{IR}, F, \Delta_{f}^{2}}^{(\gamma D)}$ & $\gamma_{\mathrm{IR}, F, \Delta_{f}^{3}}^{(\gamma D)}$ \\
\hline 8 & -0.377 & -0.446 & -0.481 \\
9 & -0.332 & -0.386 & -0.411 \\
10 & -0.288 & -0.328 & -0.344 \\
11 & -0.244 & -0.273 & -0.282 \\
12 & -0.199 & -0.219 & -0.224 \\
13 & -0.155 & -0.167 & -0.169 \\
14 & -0.111 & -0.117 & -0.118 \\
15 & -0.0665 & -0.0686 & -0.0688 \\
16 & -0.02215 & -0.0224 & -0.0224 \\
\hline \hline
\end{tabular}

sign of the anomalous dimension of the operator $\bar{\psi} \sigma_{\mu \nu} \psi$ and are opposite in sign relative to the anomalous dimensions that we calculated for $\bar{\psi} \psi$ in our previous work [22,34-39].

In Tables II-VIII we list values of the anomalous dimensions $\gamma_{\mathrm{IR}}^{(\gamma D)}, \gamma_{\mathrm{IR}}^{(\gamma D D)}, \gamma_{\mathrm{IR}}^{(\gamma D D D)}, \gamma_{\mathrm{IR}}^{(\sigma)}, \gamma_{\mathrm{IR}}^{(\sigma D)}, \gamma_{\mathrm{IR}}^{(\sigma D D)}$, and $\gamma_{\mathrm{IR}}^{(\sigma D D D)}$ for the theory with $G=\mathrm{SU}(3)$ and fermions in the fundamental representation, $R=F$, calculated to $O\left(\Delta_{f}^{p}\right)$,

TABLE III. Values of the anomalous dimension $\gamma_{\mathrm{IR}, F}^{(\gamma D D)}$ calculated to $O\left(\Delta_{f}^{p}\right)$, denoted $\gamma_{\mathrm{IR}, F, \Delta_{f}^{p}}^{(\gamma D D)}$, with $1 \leq p \leq 3$, for $G=\mathrm{SU}(3)$, as a function of $N_{f}$.

\begin{tabular}{llll}
\hline \hline$N_{f}$ & $\gamma_{\mathrm{IR}, F, \Delta_{f}}^{(\gamma D D)}$ & $\gamma_{\mathrm{IR}, F, \Delta_{f}^{2}}^{(\gamma D D)}$ & $\gamma_{\mathrm{IR}, F, \Delta_{f}^{3}}^{(\gamma D D)}$ \\
\hline 8 & -0.588 & -0.654 & -0.724 \\
9 & -0.519 & -0.570 & -0.618 \\
10 & -0.450 & -0.488 & -0.520 \\
11 & -0.381 & -0.408 & -0.427 \\
12 & -0.3115 & -0.330 & -0.340 \\
13 & -0.242 & -0.253 & -0.258 \\
14 & -0.173 & -0.179 & -0.180 \\
15 & -0.104 & -0.106 & -0.106 \\
16 & -0.0346 & -0.0348 & -0.0349 \\
\hline \hline
\end{tabular}

TABLE IV. Values of the anomalous dimension $\gamma_{\mathrm{IR}, F}^{(\gamma D D D)}$ calculated to $O\left(\Delta_{f}^{p}\right)$, denoted $\gamma_{\mathrm{IR}, F, \Delta_{f}^{p}}^{(\gamma D D D)}$, with $1 \leq p \leq 3$, for $G=\mathrm{SU}(3)$, as a function of $N_{f}$.

\begin{tabular}{llll}
\hline \hline$N_{f}$ & $\gamma_{\mathrm{IR}, F, \Delta_{f}}^{(\gamma D D D)}$ & $\gamma_{\mathrm{IR}, F, \Delta_{f}^{2}}^{(\gamma D D D)}$ & $\gamma_{\mathrm{IR}, F, \Delta_{f}^{3}}^{(\gamma D D D)}$ \\
\hline 8 & -0.739 & -0.794 & -0.900 \\
9 & -0.652 & -0.695 & -0.7675 \\
10 & -0.565 & -0.598 & -0.645 \\
11 & -0.478 & -0.501 & -0.530 \\
12 & -0.391 & -0.407 & -0.422 \\
13 & -0.304 & -0.314 & -0.321 \\
14 & -0.217 & -0.222 & -0.225 \\
15 & -0.130 & -0.132 & -0.133 \\
16 & -0.0435 & -0.0437 & -0.0437 \\
\hline \hline
\end{tabular}

TABLE V. Values of the anomalous dimension $\gamma_{\text {IR, }, F}^{(\sigma)}$ calculated to $O\left(\Delta_{f}^{p}\right)$, denoted $\gamma_{\mathrm{IR}, F, \Delta_{f}^{p}}^{(\sigma)}$, with $1 \leq p \leq 3$, for $G=\mathrm{SU}(3)$, as a function of $N_{f}$.

\begin{tabular}{llll}
\hline \hline$N_{f}$ & $\gamma_{\mathrm{IR}, F, \Delta_{f}}^{(\sigma)}$ & $\gamma_{\mathrm{IR}, F, \Delta_{f}^{2}}^{(\sigma)}$ & $\gamma_{\mathrm{IR}, F, \Delta_{f}^{3}}^{(\sigma)}$ \\
\hline 8 & -0.141 & -0.223 & -0.207 \\
9 & -0.125 & -0.188 & -0.1775 \\
10 & -0.108 & -0.156 & -0.149 \\
11 & -0.0914 & -0.125 & -0.121 \\
12 & -0.0748 & -0.0976 & -0.0953 \\
13 & -0.05815 & -0.07195 & -0.0709 \\
14 & -0.0415 & -0.0486 & -0.0482 \\
15 & -0.0249 & -0.0275 & -0.0274 \\
16 & -0.00831 & -0.00859 & -0.00859 \\
\hline \hline
\end{tabular}

denoted $\gamma_{\mathrm{IR}, F, \Delta_{f}^{p}}^{(\gamma D)}$, etc., with $p=1,2,3$, as functions of $N_{f}$ for a relevant range of $N_{f}$ values extending downward from the upper end of the conformal regime at $N_{f}=N_{u}$ (i.e., $\Delta_{f}=0$ ) within this conformal window [61]. The numbers in Table $\mathrm{V}$ are evaluations of our analytic results given in [36] and are included for comparison.

TABLE VI. Values of the anomalous dimension $\gamma_{\mathrm{IR}, F}^{(\sigma D)}$ calculated to $O\left(\Delta_{f}^{p}\right)$, denoted $\gamma_{\mathrm{IR}, F, \Delta_{f}^{p}}^{(\sigma D)}$, with $1 \leq p \leq 3$, for $G=\mathrm{SU}(3)$, as a function of $N_{f}$.

\begin{tabular}{llll}
\hline \hline$N_{f}$ & $\gamma_{\mathrm{IR}, F, \Delta_{f}}^{(\sigma D)}$ & $\gamma_{\mathrm{IR}, F, \Delta_{f}^{2}}^{(\sigma D)}$ & $\gamma_{\mathrm{IR}, F, \Delta_{f}^{3}}^{(\sigma D)}$ \\
\hline 8 & -0.424 & -0.503 & -0.527 \\
9 & -0.374 & -0.436 & -0.452 \\
10 & -0.324 & -0.3705 & -0.381 \\
11 & -0.274 & -0.307 & -0.314 \\
12 & -0.224 & -0.247 & -0.250 \\
13 & -0.174 & -0.188 & -0.190 \\
14 & -0.125 & -0.131 & -0.132 \\
15 & -0.0748 & -0.0772 & -0.0774 \\
16 & -0.0249 & -0.0252 & -0.0252 \\
\hline \hline
\end{tabular}


TABLE VII. Values of the anomalous dimension $\gamma_{\mathrm{IR}, F}^{(\sigma D D)}$ calculated to $O\left(\Delta_{f}^{p}\right)$, denoted $\gamma_{\mathrm{IR}, F, \Delta_{f}^{p}}^{(\sigma D D)}$, with $1 \leq p \leq 3$, for $G=\mathrm{SU}(3)$, as a function of $N_{f}$.

\begin{tabular}{llll}
\hline \hline$N_{f}$ & $\gamma_{\mathrm{IR}, F, \Delta_{f}}^{(\sigma D D)}$ & $\gamma_{\mathrm{IR}, F, \Delta_{f}^{2}}^{(\sigma D D)}$ & $\gamma_{\mathrm{IR}, F, \Delta_{f}^{3}}^{(\sigma D D)}$ \\
\hline 8 & -0.612 & -0.682 & -0.748 \\
9 & -0.540 & -0.594 & -0.640 \\
10 & -0.468 & -0.509 & -0.539 \\
11 & -0.396 & -0.425 & -0.443 \\
12 & -0.324 & -0.344 & -0.353 \\
13 & -0.252 & -0.264 & -0.268 \\
14 & -0.180 & -0.186 & -0.188 \\
15 & -0.108 & -0.110 & -0.111 \\
16 & -0.0360 & -0.03624 & -0.03625 \\
\hline \hline
\end{tabular}

TABLE VIII. Values of the anomalous dimension $\gamma_{\mathrm{IR}, F}^{(\sigma D D D)}$ calculated to $O\left(\Delta_{f}^{p}\right)$, denoted $\gamma_{\mathrm{IR}, F, \Delta_{f}^{p}}^{(\sigma D D D)}$, with $1 \leq p \leq 3$, for $G=\mathrm{SU}(3)$, as a function of $N_{f}$.

\begin{tabular}{llll}
\hline \hline$N_{f}$ & $\gamma_{\mathrm{IR}, F, \Delta_{f}}^{(\sigma D D D)}$ & $\gamma_{\mathrm{IR}, F, \Delta_{f}^{2}}^{(\sigma D D D)}$ & $\gamma_{\mathrm{IR}, F, \Delta_{f}^{3}}^{(\sigma D D D)}$ \\
\hline 8 & -0.753 & -0.811 & -0.913 \\
9 & -0.665 & -0.709 & -0.779 \\
10 & -0.576 & -0.610 & -0.655 \\
11 & -0.487 & -0.511 & -0.539 \\
12 & -0.399 & -0.415 & -0.430 \\
13 & -0.310 & -0.320 & -0.327 \\
14 & -0.222 & -0.2265 & -0.229 \\
15 & -0.133 & -0.135 & -0.135 \\
16 & -0.0443 & -0.0445 & -0.0445 \\
\hline \hline
\end{tabular}

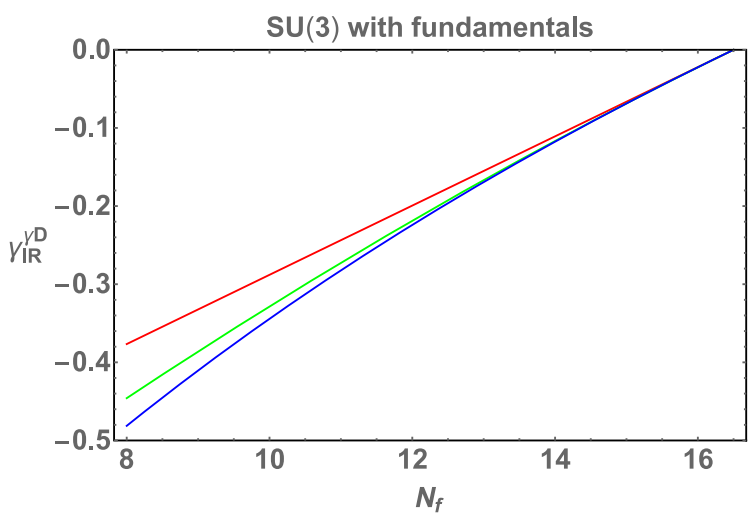

FIG. 1. Plot of the anomalous dimension $\gamma_{\mathrm{IR}, F}^{(\gamma D)}$ of the operator $\bar{\psi} \gamma \gamma_{\mu_{1}} D_{\mu_{2}} \psi$ at the IRFP for the theory with $G=\mathrm{SU}(3)$, and $N_{f}$ fermions in the fundamental representation, calculated to order $O\left(\Delta_{f}^{p}\right)$, where $p=1,2,3$. Denoting the anomalous dimension calculated to order $O\left(\Delta_{f}^{p}\right)$ as $\gamma_{\mathrm{IR}, F, \Delta_{f}^{p}}^{(\gamma D)}$, the curves, from top to bottom, refer to $\gamma_{\mathrm{IR}, F, \Delta_{f}}^{(\gamma D)}(\mathrm{red}), \gamma_{\mathrm{IR}, F, \Delta_{f}^{2}}^{(\gamma D)}$ (green), and $\gamma_{\mathrm{IR}, F, \Delta_{f}^{3}}^{(\gamma D)}$ (blue).

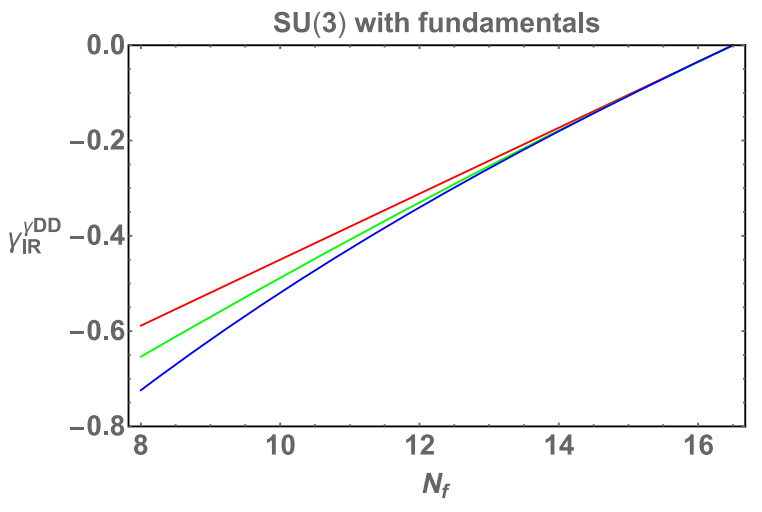

FIG. 2. Plot of the anomalous dimension $\gamma_{\mathrm{IR}, F}^{(\gamma D D)}$ of the operator $\bar{\psi} \gamma_{\mu_{1}} D_{\mu_{2}} D_{\mu_{3}} \psi$ at the IRFP for $G=\mathrm{SU}(3)$, and $N_{f}$ fermions in the fundamental representation, calculated to order $O\left(\Delta_{f}^{p}\right)$, where $p=1,2,3$. Denoting the anomalous dimension calculated to order $O\left(\Delta_{f}^{p}\right)$ as $\gamma_{\mathrm{IR}, F, \Delta_{f}^{p}}^{(\gamma D D)}$, the curves, from top to bottom, refer to $\gamma_{\mathrm{IR}, F, \Delta_{f}}^{(\gamma D D)}\left(\right.$ red), $\gamma_{\mathrm{IR}, F, \Delta_{f}^{2}}^{(\gamma D D)}$ (green), and $\gamma_{\mathrm{IR}, F, \Delta_{f}^{3}}^{(\gamma D D)}$ (blue).

In Figs. 1-7 we show plots of these anomalous dimensions for the $\mathrm{SU}(3)$ theory with $R=F$. The plot of the anomalous dimension for $\bar{\psi} \sigma_{\lambda \mu_{1}} \psi$ is based on the analytic results of our earlier paper [36] but was not given there and is new here. As can be seen from these tables and figures, the higher-order terms in the $\Delta_{f}$ expansion are sufficiently small that it is expected to be reliable throughout much of the non-Abelian Coulomb phase (i.e., conformal window). As is obvious, since our calculations are finite series expansions in powers of $\Delta_{f}$, they are most accurate in the upper part of the NACP, where this expansion parameter $\Delta_{f}$ is small. This is similar to what we found in our earlier scheme-independent calculations of anomalous dimensions

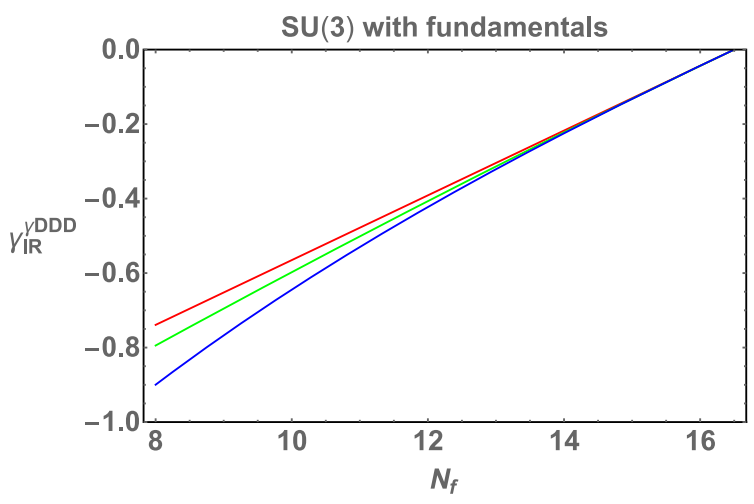

FIG. 3. Plot of the anomalous dimension $\gamma_{\mathrm{IR}, F}^{(\gamma D D D)}$ of the operator $\bar{\psi} \gamma_{\mu_{1}} D_{\mu_{2}} D_{\mu_{3}} D_{\mu_{4}} \psi$ at the IRFP for $G=\mathrm{SU}(3)$, and $N_{f}$ fermions in the fundamental representation, calculated to order $O\left(\Delta_{f}^{p}\right)$, where $p=1,2,3$. Denoting the calculation to order $O\left(\Delta_{f}^{p}\right)$ as $\gamma_{\mathrm{IR}, F, \Delta_{f}^{p}}^{(\gamma D D D)}$, from top to bottom, the colors refer to $\gamma_{\mathrm{IR}, F, \Delta_{f}}^{(\gamma D D D)}$ (red), $\gamma_{\mathrm{IR}, F, \Delta_{f}^{2}}^{(\gamma D D D)}$ (green), and $\gamma_{\mathrm{IR}, F, \Delta_{f}^{3}}^{(\gamma D D D)}$ (blue). 


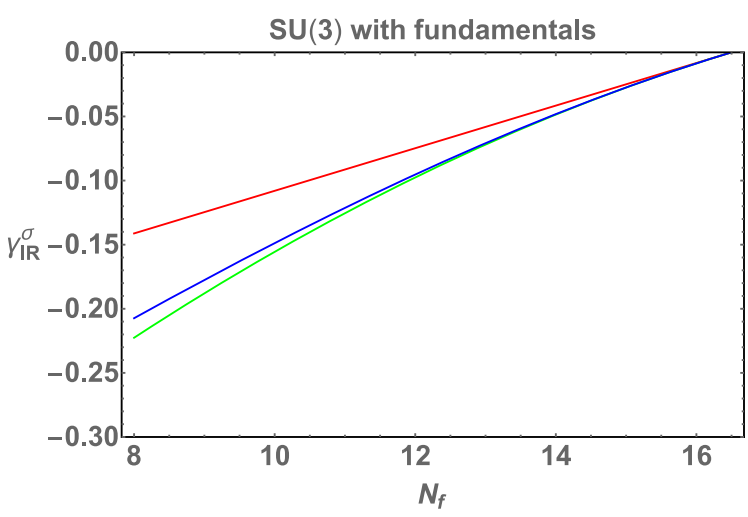

FIG. 4. Plot of the anomalous dimension $\gamma_{\mathrm{IR}, F}^{(\sigma)}$ of the operator $\bar{\psi} \sigma_{\lambda \mu_{1}} \psi$ at the IRFP for $G=\mathrm{SU}(3)$, and $N_{f}$ fermions in the fundamental representation, calculated to order $O\left(\Delta_{f}^{p}\right)$, where $p=1,2,3$. Denoting the calculation to order $O\left(\Delta_{f}^{p}\right)$ as $\gamma_{\mathrm{IR}, F, \Delta_{f}^{p}}^{(\sigma)}$, the colors refer to $\gamma_{\mathrm{IR}, F, \Delta_{f}}^{(\sigma)}$ (red), $\gamma_{\mathrm{IR}, F, \Delta_{f}^{2}}^{(\sigma)}$ (green), and $\gamma_{\mathrm{IR}, F, \Delta_{f}^{3}}^{(\sigma)}$ (blue).

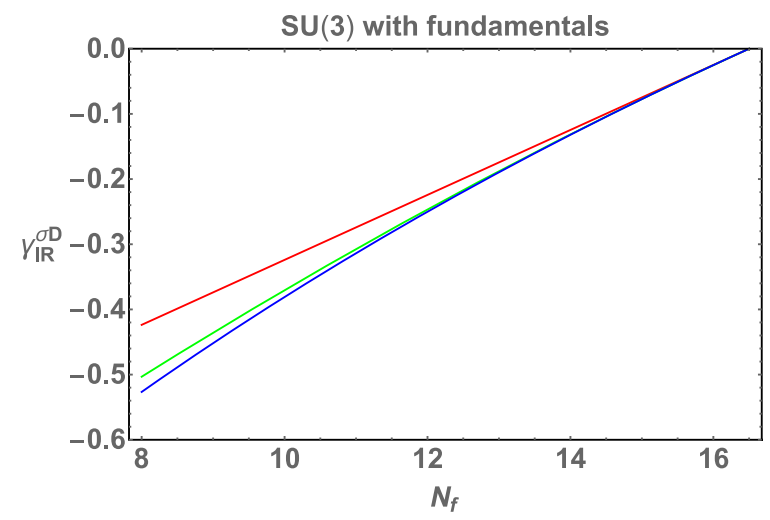

FIG. 5. Plot of the anomalous dimension $\gamma_{\text {IR,F }}^{(\sigma D)}$ of the operator $\bar{\psi} \sigma_{\lambda \mu_{1}} D_{\mu_{2}} \psi$ at the IRFP for $G=\mathrm{SU}(3)$, and $N_{f}$ fermions in the fundamental representation, calculated to order $O\left(\Delta_{f}^{p}\right)$, where $p=1,2,3$. Denoting the calculation to order $O\left(\Delta_{f}^{p}\right)$ as $\gamma_{\mathrm{IR}, F, \Delta_{f}^{p}}^{(\sigma D)}$, from top to bottom, the colors refer to $\gamma_{\mathrm{IR}, F, \Delta_{f}}^{(\sigma D)}$ (red), $\gamma_{\mathrm{IR}, F, \Delta_{f}^{2}}^{(\sigma D)}$ (green), and $\gamma_{\mathrm{IR}, F, \Delta_{f}^{3}}^{(\sigma D)}$ (blue).

[34-39]. In the figures, this is evident from the fact that the curves for the anomalous dimensions calculated to $O\left(\Delta_{f}^{3}\right)$ are reasonably close to the corresponding curves for these anomalous dimensions calculated to order $O\left(\Delta_{f}^{2}\right)$. As is evident from the values of $\gamma_{\mathrm{IR}, F, \Delta_{f}^{p}}^{(\mathcal{O})}$ that we have listed for the various operators $\mathcal{O}$ in Tables II-VIII, the fractional differences $R_{3,2}^{(\mathcal{O})} \equiv\left(\gamma_{\mathrm{IR}, F, \Delta_{f}^{3}}^{(\mathcal{O})}-\gamma_{\mathrm{IR}, F, \Delta_{f}^{2}}^{(\mathcal{O})}\right) / \gamma_{\mathrm{IR}, F, \Delta_{f}^{3}}^{(\mathcal{O})}$ are $\sim O\left(10^{-3}\right)$ for $N_{f}$ values near the upper end of the conformal window and increase as $N_{f}$ decreases; at

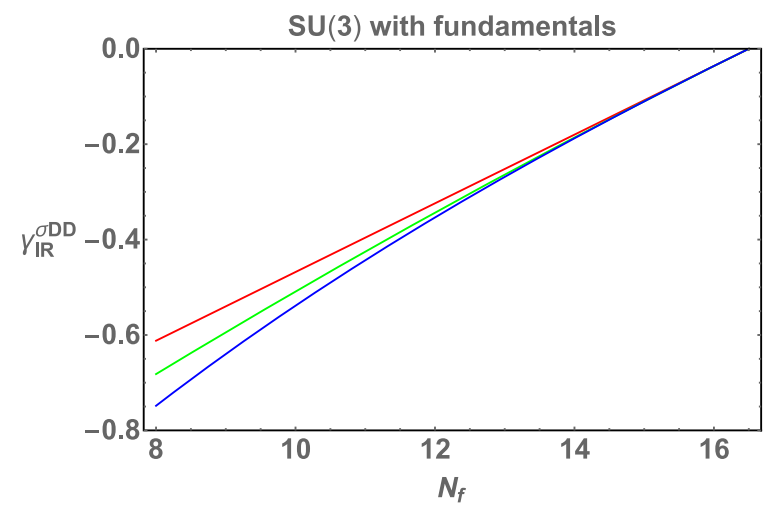

FIG. 6. Plot of the anomalous dimension $\gamma_{\mathrm{IR}, F}^{(\sigma D D)}$ of the operator $\bar{\psi} \sigma_{\lambda \mu_{1}} D_{\mu_{2}} D_{\mu_{3}} \psi$ at the IRFP for $G=\mathrm{SU}(3)$, and $N_{f}$ fermions in the fundamental representation, calculated to order $O\left(\Delta_{f}^{p}\right)$, where $p=1,2,3$. Denoting the calculation to order $O\left(\Delta_{f}^{p}\right)$ as $\gamma_{\mathrm{IR}, F, \Delta_{f}^{p}}^{(\sigma D D)}$, from top to bottom, the colors refer to $\gamma_{\mathrm{IR}, F, \Delta_{f}}^{(\sigma D D)}$ (red), $\gamma_{\mathrm{IR}, F, \Delta_{f}^{2}}^{(\sigma D D)}$ (green), and $\gamma_{\mathrm{IR}, F, \Delta_{f}^{3}}^{(\sigma D D)}$ (blue).

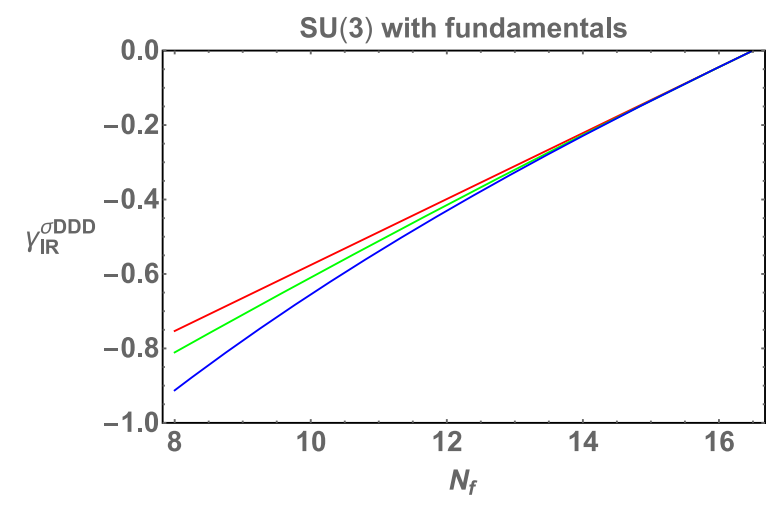

FIG. 7. Plot of the anomalous dimension $\gamma_{\mathrm{IR}, F}^{(\sigma D D D)}$ of the operator $\bar{\psi} \sigma_{\lambda \mu_{1}} D_{\mu_{2}} D_{\mu_{2}} D_{\mu_{3}} \psi$ at the IRFP for $G=\mathrm{SU}(3)$, and $N_{f}$ fermions in the fundamental representation, calculated to order $O\left(\Delta_{f}^{p}\right)$, where $p=1,2,3$. Denoting the calculation to order $O\left(\Delta_{f}^{p}\right)$ as $\gamma_{\mathrm{IR}, F, \Delta_{f}^{p}}^{(\sigma D D D)}$, from top to bottom, the colors refer to $\gamma_{\mathrm{IR}, F, \Delta_{f}}^{(\sigma D D)}$ (red), $\gamma_{\mathrm{IR}, F, \Delta_{f}^{2}}^{(\sigma D D D)}$ (green), and $\gamma_{\mathrm{IR}, F, \Delta_{f}^{3}}^{(\sigma D D)}$ (blue).

$N_{f}=12$, the $R_{3,2}^{(\mathcal{O})}$ are a few per cent, and at $N_{f}=8$, $R_{3,2}^{(\mathcal{O})} \sim 0.1$. For a given $N_{f}, R_{3,2}^{(\mathcal{O})}$ increases slightly with the spin of the operator $\mathcal{O}$; for example, for $N_{f}=12$, $R_{3,2}^{(\gamma D)}=0.024, R_{3,2}^{(\gamma D D)}=0.032$, and $R_{3,2}^{(\gamma D D D)}=0.037$, while $R_{3,2}^{(\sigma D)}=0.014, R_{3,2}^{(\sigma D D)}=0.028$, and $R_{3,2}^{(\sigma D D D)}=0.035$.

\section{LNN LIMIT FOR $\gamma_{\text {IR,SU }\left(N_{c}\right), F}^{(\mathcal{O})}$}

In a theory with gauge group $\mathrm{SU}\left(N_{c}\right)$ and fermions in the fundamental representation, $R=F$, it is of interest to consider the limit 
$N_{c} \rightarrow \infty, \quad N_{F} \rightarrow \infty \quad$ with $\quad r \equiv \frac{N_{F}}{N_{c}}$ fixed and finite and $\xi(\mu) \equiv \alpha(\mu) N_{c}$ is a finite function of $\mu$.

This limit is denoted as $\lim _{\mathrm{LNN}}$ [where "LNN" connotes "large $N_{c}$ and $N_{F}$ " with the constraints in Eq. (6.1) imposed]. It is also often called the 't Hooft-Veneziano limit. It has the simplifying feature that rather than depending on $N_{c}$ and $N_{f}$, the properties of the theory only depend on their ratio, $r$. The scheme-independent expansion parameter in this LNN limit is

$$
\begin{gathered}
\Delta_{r} \equiv \lim _{\mathrm{LNN}} \frac{\Delta_{f}}{N_{c}}=\frac{11}{2}-r . \\
r_{u}=\lim _{\mathrm{LNN}} \frac{N_{u}}{N_{c}},
\end{gathered}
$$

and

$$
r_{\ell}=\lim _{\mathrm{LNN}} \frac{N_{\ell}}{N_{c}}
$$

with values

$$
r_{u}=\frac{11}{2}=5.5
$$

and

$$
r_{\ell}=\frac{34}{13}=2.6154 \text {. }
$$

With $I_{\text {IRZ }}: N_{\ell}<N_{f}<N_{u}$, it follows that the corresponding interval in the ratio $r$ is

$$
I_{\mathrm{IRZ}, r}: \frac{34}{13}<r<\frac{11}{2} \text {, i.e., } 2.6154<r<5.5 .
$$

Here we evaluate these scheme-independent anomalous dimension coefficients in a theory with $G=\mathrm{SU}\left(N_{c}\right)$ and $R=F$, in the LNN limit. The rescaled coefficients that are finite in the LNN limit are

$$
\hat{\kappa}_{n}^{(\mathcal{O})}=\lim _{N_{c} \rightarrow \infty} N_{c}^{n} \kappa_{n}^{(\mathcal{O})} .
$$

The anomalous dimension $\gamma_{\mathrm{IR}}^{(\mathcal{O})}$ is also finite in this limit and is given by

$$
\lim _{\mathrm{LNN}} \gamma_{\mathrm{IR}, \mathrm{SU}\left(N_{c}\right), F}^{(\mathcal{O})}=\sum_{n=1}^{\infty} \kappa_{n}^{(\mathcal{O})} \Delta_{f}^{n}=\sum_{n=1}^{\infty} \hat{\kappa}_{n}^{(\mathcal{O})} \Delta_{r}^{n} .
$$

As $r$ decreases from its upper limit, $r_{u}$, to $r_{\ell}$, the expansion variable $\Delta_{r}$ increases from 0 to

$$
\left(\Delta_{r}\right)_{\max }=\frac{75}{26}=2.8846 \quad \text { for } r \in I_{\mathrm{IRZ}, r} .
$$

In this LNN limit, the values of $\hat{\kappa}_{n}^{(\mathcal{O})}$ with $1 \leq n \leq 3$ for the operators $\mathcal{O}$ considered here are listed in Table IX. For comparison, we also include the corresponding values of
TABLE IX. Values of the $\hat{\kappa}_{n}^{(\mathcal{O})}$ coefficients for $G=\operatorname{SU}\left(N_{c}\right)$ and $R=F$ in the LNN limit. The operators are indicated by their shorthand symbols, so 1 refers to $\bar{\psi} \psi ; \sigma$ refers to $\bar{\psi} \sigma_{\lambda \mu_{1}} \psi ; \gamma D$ to $\bar{\psi} \gamma \gamma_{\mu_{1}} D_{\mu_{2}} \psi$, etc. The notation $a \mathrm{e}-n$ means $a \times 10^{-n}$.

\begin{tabular}{llll}
\hline \hline $\mathcal{O}$ & \multicolumn{1}{c}{$\hat{\kappa}_{1}^{(\mathcal{O})}$} & \multicolumn{1}{c}{$\hat{\kappa}_{2}^{(\mathcal{O})}$} & $\hat{\kappa}_{3}^{(\mathcal{O})}$ \\
\hline 1 & 0.160000 & 0.0376320 & $0.832074 \mathrm{e}-2$ \\
$\sigma$ & -0.0533333 & $-0.969956 \mathrm{e}-2$ & $2.33189 \mathrm{e}-4$ \\
$\gamma D$ & -0.142222 & $-1.04844 \mathrm{e}-2$ & $-2.135375 \mathrm{e}-3$ \\
$\gamma D D$ & -0.222222 & $-1.11967 \mathrm{e}-2$ & $-0.404507 \mathrm{e}-2$ \\
$\gamma D D D$ & -0.279111 & $-1.08149 \mathrm{e}-2$ & $-0.572019 \mathrm{e}-2$ \\
$\sigma D$ & -0.160000 & -0.0120320 & $-1.967385 \mathrm{e}-3$ \\
$\sigma D D$ & -0.231111 & $-1.17960 \mathrm{e}-2$ & $-0.397447 \mathrm{e}-2$ \\
$\sigma D D D$ & -0.284444 & $-1.114495 \mathrm{e}-2$ & $-0.567795 \mathrm{e}-2$ \\
\hline \hline
\end{tabular}

$\hat{\kappa}_{n}^{(\mathcal{O})}$ for the operators $\bar{\psi} \psi$ and $\bar{\psi} \sigma_{\mu \nu} \psi$ that we had calculated in [36].

\section{EVALUATION OF ANOMALOUS DIMENSIONS $\gamma_{\mathrm{IR}}^{(\mathcal{O})}$ FOR $G=\mathrm{SU}\left(N_{c}\right)$ AND $R=A d j$}

For the case where $G=\mathrm{SU}\left(N_{c}\right)$ and the fermions are in the adjoint representation, $R=A d j$, our general results for the scheme-independent expansion coefficients for the anomalous dimensions of the operators under consideration are as follows::

$$
\begin{aligned}
& \kappa_{1, \mathrm{SU}\left(N_{c}\right), \operatorname{Adj}}^{(\gamma D)}=-\frac{2^{5}}{3^{4}}=-0.395062, \\
& \kappa_{2, \mathrm{SU}\left(N_{c}\right), A d j}^{(\gamma D)}=-\frac{1756}{3^{9}}=-0.0892140, \\
& \kappa_{3, \mathrm{SU}\left(N_{c}\right), \operatorname{Adj}}^{(\gamma D)}=-\frac{88129}{3^{14}}+\frac{4736}{3^{10} N_{c}^{2}} \\
& =-0.0184256+\frac{0.0802046}{N_{c}^{2}}, \\
& \kappa_{1, \mathrm{SU}\left(N_{c}\right), \operatorname{Adj}}^{(\gamma D D)}=-\frac{50}{3^{4}}=-0.617284, \\
& \kappa_{2, \mathrm{SU}\left(N_{c}\right), \mathrm{Adj}}^{(\gamma D D)}=-\frac{10165}{2^{2} \cdot 3^{9}}=-0.129109, \\
& \kappa_{3, \mathrm{SU}\left(N_{c}\right), \operatorname{Adj}}^{(\gamma D D)}=-\frac{2272255}{2^{4} \cdot 3^{14}}+\frac{7400}{3^{10} N_{c}^{2}} \\
& =-0.0296920+\frac{0.125320}{N_{c}^{2}} \text {, } \\
& \kappa_{1, \mathrm{SU}\left(N_{c}\right), A d j}^{(\gamma D D D)}=-\frac{314}{3^{4} \cdot 5}=-0.775309, \\
& \kappa_{2, \mathrm{SU}\left(N_{c}\right), A d j}^{(\gamma D D)}=-\frac{1504769}{2^{2} \cdot 3^{9} \cdot 5^{3}}=-0.152900,
\end{aligned}
$$


and

$$
\begin{array}{r}
\kappa_{3, \mathrm{SU}\left(N_{c}\right), A d j}^{(\gamma D D)}=-\frac{9206650603}{2^{4} \cdot 3^{14} \cdot 5^{5}}+\frac{46472}{3^{10} \cdot 5 N_{c}^{2}} \\
=-0.0384976+\frac{0.1574015}{N_{c}^{2}}, \\
\kappa_{1, \mathrm{SU}\left(N_{c}\right), A d j}^{(\sigma D)}=-\frac{2^{2}}{3^{2}}=-0.444444, \\
\kappa_{2, \mathrm{SU}\left(N_{c}\right), A d j}^{(\sigma D)}=-\frac{149}{2 \cdot 3^{6}}=-0.102195,
\end{array}
$$

and

$$
\begin{array}{r}
\kappa_{3, \mathrm{SU}\left(N_{c}\right), \mathrm{Adj}}^{(\sigma D)}=-\frac{10801}{2^{3} \cdot 3^{10}}+\frac{592}{3^{8} N_{c}^{2}} \\
=-0.0228645+\frac{0.0902301}{N_{c}^{2}}, \\
\kappa_{1, \mathrm{SU}\left(N_{c}\right), A d j}^{(\sigma D D)}=-\frac{52}{3^{4}}=-0.641975, \\
\kappa_{2, \mathrm{SU}\left(N_{c}\right), A d j}^{(\sigma D D)}=-\frac{5123}{2 \cdot 3^{9}}=-0.130138, \\
\kappa_{3, \mathrm{SU}\left(N_{c}\right), \operatorname{Adj}}^{(\sigma D D)}=-\frac{984949}{2^{3} \cdot 3^{14}}+\frac{7696}{3^{10} N_{c}^{2}} \\
=-0.0257410+\frac{0.130332}{N_{c}^{2}}, \\
\kappa_{1, \mathrm{SU}\left(N_{c}\right), A d j}^{(\sigma D D D)}=-\frac{2^{6}}{3^{4}}=-0.790123, \\
\kappa_{2, \mathrm{SU}\left(N_{c}\right), \operatorname{Adj} j}^{(\sigma D D D)}=-\frac{3070}{3^{9}}=-0.155972
\end{array}
$$

and

$$
\begin{aligned}
\kappa_{3, \mathrm{SU}\left(N_{c}\right), A d j}^{(\sigma D D D)} & =-\frac{378247}{2 \cdot 3^{14}}+\frac{9472}{3^{10} N_{c}^{2}} \\
& =-0.0395410+\frac{0.160409}{N_{c}^{2}} .
\end{aligned}
$$

For all of these operators $\mathcal{O}$, the coefficients $\kappa_{n, \mathrm{SU}\left(N_{c}\right), \operatorname{Adj}}^{(\mathcal{O})}$ are negative for $n=1$ and $n=2$ and for all $N_{c}$. The coefficient $\kappa_{3, \mathrm{SU}\left(N_{c}\right), A d j}^{(\sigma D)}$ is negative for all $N_{c}$, while the coefficients $\kappa_{3, \mathrm{SU}\left(N_{c}\right), A d j}^{(\mathcal{O})}$ for the other operators are positive for $N_{c}=2$, i.e., $G=\mathrm{SU}(2)$, and are negative for $N_{c} \geq 3$.

\section{CONCLUSIONS}

In conclusion, in this paper we have calculated schemeindependent expansions up to $O\left(\Delta_{f}^{3}\right)$ inclusive for the anomalous dimensions of the higher-spin, twist-2 bilinear fermion operators $\bar{\psi} \gamma_{\mu_{1}} D_{\mu_{2}} \ldots D_{\mu_{j}} \psi$ and $\bar{\psi} \sigma_{\lambda \mu_{1}} D_{\mu_{2}} \ldots D_{\mu_{j}} \psi$ with $j$ up to 3, evaluated at an IR fixed point in the nonAbelian Coulomb phase of an asymptotically free gauge theory with gauge group $G$ and $N_{f}$ fermions transforming according to a representation $R$ of $G$. Our general results are evaluated for several special cases, including the case $G=\mathrm{SU}\left(N_{c}\right)$ with $R$ equal to the fundamental and adjoint representations. We have presented our results in convenient tabular and graphical formats. For fermions in the fundamental representation, we also analyze the limit $N_{c} \rightarrow$ $\infty$ and $N_{f} \rightarrow \infty$ with $N_{f} / N_{c}$ fixed and finite. A comparison with our previous scheme-independent calculations of the corresponding anomalous dimensions of $\bar{\psi} \psi$ and $\bar{\psi} \sigma_{\mu \nu} \psi$ has also been given. Our new results further elucidate the properties of conformal field theories. With the requisite inputs, one could extend these scheme-independent calculations to higher-spin operators and to higher order in powers of $\Delta_{f}$. It is hoped that lattice measurements of these anomalous dimensions of higher-spin operators in the conformal window will be performed in the future, and it will be of interest to compare our calculations with lattice results when they will become available.

\section{ACKNOWLEDGMENTS}

We thank J. Gracey for discussions. This research was supported in part by the Danish National Research Foundation grant DNRF90 to $\mathrm{CP}^{3}$-Origins at SDU (T. A. R.) and by the U.S. NSF Grants NSF-PHY1620628 and NSF-PHY-1915093 (R. S.).

\section{APPENDIX: PREVIOUS RESULTS ON $\gamma^{(1)}$ AND $\gamma^{(\sigma)}$ FOR $G=\mathrm{SU}(3)$ AND $R=F$}

In this appendix, for comparison with our new results, we list our previous results from [36] (see also [37]) for the scheme-independent series expansions of the anomalous dimensions $\gamma_{\mathrm{IR}}^{(\mathcal{O})}$ for $\mathcal{O}=\bar{\psi} \psi$ and $\mathcal{O}=\bar{\psi} \sigma_{\mu \nu} \psi$. Following the same shorthand notation as in the text, we denote the coefficients at order $O\left(\Delta_{f}^{n}\right)$ in the scheme-independent series expansions (2.10) for these anomalous dimensions as $\kappa_{n}^{(1)}$ and $\kappa_{n}^{(\sigma)}$. We calculated

$$
\kappa_{1}^{(1)}=\frac{8 T_{f} C_{f}}{C_{A} D},
$$

$$
\kappa_{2}^{(1)}=\frac{4 T_{f}^{2} C_{f}\left(5 C_{A}+88 C_{f}\right)\left(7 C_{A}+4 C_{f}\right)}{3 C_{A}^{2} D^{3}},
$$




$$
\begin{aligned}
\kappa_{3}^{(1)}= & \frac{4 T_{f} C_{f}}{3^{4} C_{A}^{4} D^{5}}\left[-55419 T_{f}^{2} C_{A}^{5}+432012 T_{f}^{2} C_{A}^{4} C_{f}+5632 T_{f}^{2} C_{f} \frac{d_{A}^{a b c d} d_{A}^{a b c d}}{d_{A}}\left(-5+132 \zeta_{3}\right)\right. \\
& +16 C_{A}^{3}\left(122043 T_{f}^{2} C_{f}^{2}+6776 \frac{d_{R}^{a b c d} d_{R}^{a b c d}}{d_{A}}\left(-11+24 \zeta_{3}\right)\right) \\
& +704 C_{A}^{2}\left(1521 T_{f}^{2} C_{f}^{3}+112 T_{f} \frac{d_{R}^{a b c d} d_{A}^{a b c d}}{d_{A}}\left(4-39 \zeta_{3}\right)+242 C_{f} \frac{d_{R}^{a b c d} d_{R}^{a b c d}}{d_{A}}\left(-11+24 \zeta_{3}\right)\right) \\
& \left.+32 T_{f} C_{A}\left(53361 T_{f} C_{f}^{4}-3872 C_{f} \frac{d_{R}^{a b c d} d_{A}^{a b c d}}{d_{A}}\left(-4+39 \zeta_{3}\right)+112 T_{f} \frac{d_{A}^{a b c d} d_{A}^{a b c d}}{d_{A}}\left(-5+132 \zeta_{3}\right)\right)\right]
\end{aligned}
$$

where the denominator factor $D$ was defined in Eq. (3.1)]. In [37,39] we presented results for the next-higher order coefficient, $\kappa_{4}^{(1)}$, but these are not needed here.

For the $\kappa_{n}^{(\sigma)}$ we found

$$
\begin{gathered}
\kappa_{1}^{(\sigma)}=-\frac{8 C_{f} T_{f}}{3 C_{A} D} \\
\kappa_{2}^{(\sigma)}=-\frac{4 C_{f} T_{f}^{2}\left(259 C_{A}^{2}+428 C_{A} C_{f}-528 C_{f}^{2}\right)}{9 C_{A}^{2} D^{3}}
\end{gathered}
$$

and

$$
\begin{aligned}
\kappa_{3}^{(\sigma)}= & \frac{4 C_{f} T_{f}}{3^{5} C_{A}^{4} D^{5}}\left[3 C _ { A } T _ { f } ^ { 2 } \left\{C_{A}^{4}\left(-11319+188160 \zeta_{3}\right)+C_{A}^{3} C_{f}\left(-337204+64512 \zeta_{3}\right)+C_{A}^{2} C_{f}^{2}\left(83616-890112 \zeta_{3}\right)\right.\right. \\
& \left.+C_{A} C_{f}^{3}\left(1385472-354816 \zeta_{3}\right)+C_{f}^{4}\left(-212960+743424 \zeta_{3}\right)\right\}-512 T_{f}^{2} D\left(-5+132 \zeta_{3}\right) \frac{d_{A}^{a b c d} d_{A}^{a b c d}}{d_{A}} \\
& \left.-15488 C_{A}^{2} D\left(-11+24 \zeta_{3}\right) \frac{d_{R}^{a b c d} d_{R}^{a b c d}}{d_{A}}+11264 C_{A} T_{f} D\left(-4+39 \zeta_{3}\right) \frac{d_{R}^{a b c d} d_{A}^{a b c d}}{d_{A}}\right] .
\end{aligned}
$$

For $G=\mathrm{SU}\left(N_{c}\right)$ and $R=F$, in the LNN limit, these yield the rescaled coefficients

$$
\begin{gathered}
\hat{\kappa}_{1}^{(1)}=\frac{4}{5^{2}}=0.1600, \\
\hat{\kappa}_{2}^{(1)}=\frac{588}{5^{6}}=0.037632, \\
\hat{\kappa}_{3}^{(1)}=\frac{2193944}{3^{3} \cdot 5^{10}}=0.83207 \times 10^{-2},
\end{gathered}
$$

$$
\hat{\kappa}_{1}^{(\sigma)}=-\frac{4}{3 \cdot 5^{2}}=-0.053333
$$

$$
\hat{\kappa}_{2}^{(\sigma)}=-\frac{1364}{3^{2} \cdot 5^{6}}=-\left(0.969956 \times 10^{-2}\right)
$$

and

$$
\hat{\kappa}_{3}^{(\sigma)}=\frac{184456}{3^{4} \cdot 5^{10}}=2.3319 \times 10^{-4} .
$$

[1] This assumption of massless fermions does not entail any loss of generality, since a fermion with nonzero mass $m$ would be integrated out of the low-energy effective field theory that describes the physics at Euclidean momentum scales $\mu<m$ and hence would not affect the infrared limit $\mu \rightarrow 0$ that we consider here. 
[2] K. G. Wilson, Phys. Rev. 179, 1499 (1969); K. G. Wilson and W. Zimmermann, Commun. Math. Phys. 24, 87 (1972).

[3] There is an extensive literature on operator product expansions, the renormalization group, and applications to deep inelastic scattering and QCD. Some of the early papers, in addition to [2], include [4-11]; early reviews include [12-13].

[4] C. G. Callan and D. J. Gross, Phys. Rev. Lett. 22, 156 (1969); D. J. Gross and C. H. Llewellyn Smith, Nucl. Phys. B14, 337 (1969).

[5] C. G. Callan, Phys. Rev. D 2, 1541 (1970); K. Symanzik, Commun. Math. Phys. 18, 227 (1970).

[6] Y. Frishman, Phys. Rev. Lett. 25, 966 (1970).

[7] D. J. Gross and S. B. Treiman, Phys. Rev. D 4, 1059 (1971).

[8] R. A. Brandt and G. Preparata, Nucl. Phys. B27, 541 (1971).

[9] N. H. Christ, B. Hasslacher, and A. H. Mueller, Phys. Rev. D 6, 3543 (1972).

[10] D. J. Gross and F. Wilczek, Phys. Rev. Lett. 30, 1343 (1973); H. D. Politzer, Phys. Rev. Lett. 30, 1346 (1973); G. 't Hooft (unpublished).

[11] D. J. Gross and F. Wilczek, Phys. Rev. D 8, 3633 (1973); 9, 980 (1974).

[12] H. D. Politzer, Phys. Rep. 14, 129 (1974).

[13] D. J. Gross, in Methods in Field Theory, Les Houches 1975, edited by R. Balian and J. Zinn-Justin (North Holland, Amsterdam, 1976), p. 141.

[14] There are also twist- 2 operators that are bilinear in the fieldstrength tensors of the gauge field and do not directly involve the fermions. Here we focus on higher-spin bilinear fermion operators.

[15] Some of the early papers on transversity distributions include J. P. Ralston and D. E. Soper, Nucl. Phys. B152, 109 (1979); X. Artru and M. Mekhfi, Z. Phys. C 45, 669 (1990); R. L. Jaffe and X. Ji, Phys. Rev. Lett. 67, 552 (1991); W. Vogelsang and A. Weber, Phys. Rev. D 48, 2073 (1993); a recent review is C. A. Aidala, S. D. Bass, D. Hasch, and G. K. Mallot, Rev. Mod. Phys. 85, 655(2013).

[16] Some early analyses of connections between scale and conformal invariance include A. Salam, Ann. Phys. (N.Y.) 53, 174 (1969); A. M. Polyakov, JETP Lett. 12, 381 (1970); D. J. Gross and J. Wess, Phys. Rev. D 2, 753 (1970); C. G. Callan, S. Coleman, and R. Jackiw, Ann. Phys. (N.Y.) 59, 42 (1970). More recent works include J. Polchinski, Phys. B303, 226 (1988); J.-F. Fortin, B. Grinstein, and A. Stergiou, J. High Energy Phys. 01 (2013) 184; A. Dymarsky, Z. Komargodski, A. Schwimmer, and S. Thiessen, J. High Energy Phys. 10 (2015) 171, and references therein.

[17] Here and below, we will often formally generalize $N_{f}$ from a positive integer to a positive real number. If an expression such as (1.6) or (1.7) evaluates to a nonintegral real value, it is understood implicitly that one infers an appropriate integral value from it.

[18] We recall the definitions of relevant group invariants. Let $T_{a}$ denote the generators of a Lie algebra of $G$, and let $d(R)$ denote the dimension of a representation $R$ of $G$. Then the quadratic Casimir invariant $C_{2}(R)$ is defined by $\sum_{a} T_{R}^{a} T_{R}^{a}=C_{2}(R) I$, where $I$ is the $d_{R} \times d_{R}$ identity matrix. We utilize the common notation $C_{2}(A d j) \equiv C_{2}(G) \equiv C_{A}$. The trace invariant $T(R)$ is defined by $\operatorname{Tr}_{R}\left(T_{R}^{a} T_{R}^{b}\right)=$ $T(R) \delta^{a b}$. We use standard normalizations for $\mathrm{SU}\left(N_{c}\right)$ so that, e.g., for the fundamental representation $F, T(F)=1 / 2$ and $C_{2}(F)=\left(N_{c}^{2}-1\right) /\left(2 N_{c}\right)$.

[19] W. E. Caswell, Phys. Rev. Lett. 33, 244 (1974); D. R. T. Jones, Nucl. Phys. B75, 531 (1974).

[20] T. Banks and A. Zaks, Nucl. Phys. B196, 189 (1982).

[21] A. Deur, S. J. Brodsky, and G. F. de Téramond, Prog. Part. Nucl. Phys. 90, 1 (2016), and references therein.

[22] T. A. Ryttov and R. Shrock, Phys. Rev. D 83, 056011 (2011).

[23] C. Pica and F. Sannino, Phys. Rev. D 83, 035013 (2011).

[24] R. Shrock, Phys. Rev. D 87, 105005 (2013); 87, 116007 (2013).

[25] T. A. Ryttov and R. Shrock, Phys. Rev. D 94, 105015 (2016).

[26] See, e.g., S. J. Brodsky and X.-G. Wu, Phys. Rev. Lett. 109, 042002 (2012); M. Mojaza, S. J. Brodsky, and X.-G. Wu, Phys. Rev. Lett. 110, 192001 (2013); X.-G. Wu, Y. Ma, S.-Q. Wang, H.-B. Fu, H.-H. Ma, S. J. Brodsky, and M. Mojaza, Rep. Prog. Phys. 78, 126201 (2015), and references therein.

[27] T. A. Ryttov and R. Shrock, Phys. Rev. D 86, 065032 (2012); Phys. Rev. D 86, 085005 (2012).

[28] R. Shrock, Phys. Rev. D 88, 036003 (2013); 90, 045011 (2014); 91, 125039 (2015).

[29] T. Ryttov, Phys. Rev. D 89, 016013 (2014); 89, 056001 (2014); 90, 056007 (2014).

[30] J. A. Gracey and R. M. Simms, Phys. Rev. D 91, 085037 (2015).

[31] G. Choi and R. Shrock, Phys. Rev. D 90, 125029 (2014); 93, 065013 (2016); 94, 065038 (2016); G. Choi, T. A. Ryttov, and R. Shrock, Phys. Rev. D 95, 025012 (2017).

[32] G. 't Hooft, in The Whys of Subnuclear Physics, Proceedings of 1977 Erice Summer School, edited by A. Zichichi (Plenum, New York, 1979), p. 943.

[33] E. Gardi and M. Karliner, Nucl. Phys. B529, 383 (1998); E. Gardi and G. Grunberg, J. High Energy Phys. 03 (1999) 024.

[34] T. A. Ryttov, Phys. Rev. Lett. 117, 071601 (2016).

[35] T. A. Ryttov and R. Shrock, Phys. Rev. D 94, 105014 (2016).

[36] T. A. Ryttov and R. Shrock, Phys. Rev. D 94, 125005 (2016).

[37] T. A. Ryttov and R. Shrock, Phys. Rev. D 95, 085012 (2017); 95, 105004 (2017).

[38] T. A. Ryttov and R. Shrock, Phys. Rev. D 85, 076009 (2012); 96, 105018 (2017); 97, 065020 (2018).

[39] T. A. Ryttov and R. Shrock, Phys. Rev. D 96, 105015 (2017); 97, 016020 (2018); 97, 025004 (2018); 98, 096003 (2018); G. Girmohanta, T. A. Ryttov, and R. Shrock, Phys. Rev. D 99, 116022 (2019).

[40] J. A. Gracey, T. A. Ryttov, and R. Shrock, Phys. Rev. D 97, 116018 (2018).

[41] For recent reviews of relevant lattice simulations, see, e.g., Lattice for BSM 2017 Workshop, http://www-hep.colorado .edu/(tilde)eneil/lbsm17; Lattice-2017, http://wpd.ugr.es/ (tilde)lattice2017; [42]; Lattice-2018, https://web.pa .msu.edu/conf/Lattice2018; Lattice-2019, http://lattice2019 .ccnu.edu.cn.

[42] T. A. Ryttov and R. Shrock, organizers, Simons Workshop on Continuum and Lattice Approaches to the Infrared 
Behavior of Conformal and Quasiconformal Gauge Theories (2018), http://scgp.stonybrook.edu/archives/21358.

[43] As noted in the text, it is hoped that lattice measurements will be performed of the anomalous dimensions of the higher-spin bilinear fermion operators for which we have calculated scheme-independent expansions. As an example of lattice measurements that have been performed for the anomalous dimension of the spin- 0 operator $\bar{\psi} \psi$, one could take the illustrative case of $G=\mathrm{SU}(3)$ and $R=F$, for which $N_{u}=16.5$ and $N_{\ell}=8.05$, so that the value $N_{f}=12$ lies roughly midway between $N_{\ell}$ and $N_{u}$. Some lattice measurements of the anomalous dimension of $\bar{\psi} \psi$ in the IR for this $\mathrm{SU}(3)$ theory with $R=F$ and $N_{f}=12$ include T. Appelquist et al. (LSD Collaboration), Phys. Rev. D 84, 054501 (2011); T. DeGrand, Phys. Rev. D 84, 116901 (2011); Y. Aoki et al. (LatKMI Collaboration), Phys. Rev. D 86, 054506 (2012); A. Cheng, A. Hasenfratz, G. Petropoulos, and D. Schaich, J. High Energy Phys. 07 (2013) 061; A. Hasenfratz and D. Schaich, J. High Energy Phys. 10 (2018) 132; M. P. Lombardo, K. Miura, T. J. Nunes da Silva, and E. Pallante, J. High Energy Phys. 12 (2014) 183; Z. Fodor et al., Phys. Rev. D 94, 091501 (2016); Phys. Lett. B 779, 230 (2018).

[44] Some authors use the opposite sign convention for the anomalous dimension, writing $D_{\mathcal{O}}=D_{\mathcal{O} \text {,free }}+\gamma_{\mathcal{O}}$. Our sign convention is the same as the one used in the lattice gauge theory literature $[41,42]$. The equivalent notation $\gamma^{(\mathcal{O})} \equiv \gamma_{\mathcal{O}}$ will often be used.

[45] In contrast, in the confined QCD-type phase at lower $N_{f}$, although the UV beta function may exhibit a formal IR zero, as the theory flows from the UV to the IR, the running coupling becomes large enough so that spontaneous chiral symmetry breaking occurs, giving the fermions dynamical masses, so that they are integrated out of the low-energy effective field theory that is applicable deeper in the IR, and hence the resultant beta function is that of a pure gauge theory, which does not have a (perturbative) IR zero. If $N_{f}$ is only slightly less than $N_{f, c r}$, these theories thus exhibit quasiconformal behavior. If the boundary in $N_{f}$ between the NACP and the QCD-type regime involves a continuous, although nonanalytic, change in the values of anomalous dimensions, then our previous scheme-independent results in [34-39] and our results here may give some approximate guide to the values of the corresponding anomalous dimensions in quasiconformal theories with $N_{f}$ slightly less than $N_{f, c r}$.
[46] J. A. Gracey, Phys. Lett. B 488, 175 (2000).

[47] See, e.g., I. M. Gelfand, R. A. Minlos, and Z. Ya Shapiro, Representations of the Rotation and Lorentz Groups and Their Applications (Pergamon Press, New York, 1963); W.-K. Tung, Group Theory in Physics (World Scientific, Singapore, 1985).

[48] G. Mack, Commun. Math. Phys. 55, 1 (1977); B. Grinstein, K. Intriligator, and I. Rothstein, Phys. Lett. B 662, 367 (2008); Y. Nakayama, Phys. Rep. 569, 1 (2015).

[49] For a given representation $R$ of $G, d_{R}^{a b c d}=$ $(1 / 3 !) \operatorname{Tr}_{R}\left[T_{a}\left(T_{b} T_{c} T_{d}+T_{b} T_{d} T_{c}+T_{c} T_{b} T_{d}+T_{c} T_{d} T_{b}+\right.\right.$ $\left.\left.T_{d} T_{b} T_{c}+T_{d} T_{c} T_{b}\right)\right]$; see, e.g., T. van Ritbergen, A. N. Schellekens, and J. A. M. Vermaseren, Int. J. Mod. Phys. A 14, 41 (1999).

[50] O. V. Tarasov, A. A. Vladimirov, and A. Yu. Zharkov, Phys. Lett. B 93, 429 (1980); S. A. Larin and J. A. M. Vermaseren, Phys. Lett. B 303, 334 (1993).

[51] T. van Ritbergen, J. A. M. Vermaseren, and S. A. Larin, Phys. Lett. B 400, 379 (1997).

[52] K. G. Chetyrkin, Phys. Lett. B 404, 161 (1997); J. A. M. Vermaseren, S. A. Larin, and T. van Ritbergen, Phys. Lett. B 405, 327 (1997).

[53] E. G. Floratos, D. A. Ross, and C. T. Sachrajda, Nucl. Phys. B129, 66 (1977); B152, 493 (1979).

[54] J. A. Gracey, Nucl. Phys. B667, 242 (2003).

[55] J. A. Gracey, J. High Energy Phys. 10 (2006) 040.

[56] See also S. Kumano and M. Miyama, Phys. Rev. D 56, R2504 (1997); V. N. Velizhanin, Nucl. Phys. B864, 113 (2012).

[57] With our definition (2.1) [44], the inputs for our calculations of anomalous dimensions of higher-spin operators from Refs. [54,55] involve multiplication by a factor of (-2). For other operators, this procedure was already applied, for example, in Ref. [40].

[58] W. A. Bardeen, A. J. Buras, D. W. Duke, and T. Muta, Phys. Rev. D 18, 3998 (1978).

[59] F. Herzog, B. Ruijl, T. Ueda, J. A. M. Vermaseren, and A. Vogt, J. High Energy Phys. 02 (2017) 090.

[60] P. A. Baikov, K. G. Chetyrkin, and J. H. Kühn, Phys. Rev. Lett. 118, 082002 (2017).

[61] Some of the lowest values of $N_{f}$ in these tables and figures may lie below $N_{f, c r}$. However, since at present there is not a complete consensus among lattice groups concerning the value of $N_{f, c r}$ for this $\mathrm{SU}(3)$ theory with fermions in the fundamental representation, $R=F[41,42]$, we list these values for completeness. 